\title{
Solving Steiner Tree Problems in Graphs to Optimality
}

\author{
T. Koch, A. Martin \\ Konrad-Zuse-Zentrum für Informationstechnik Berlin, Takustrasse 7, D-14195 Berlin-Dahlem, \\ Germany
}

Received 11 February 1997; accepted 9 April 1998

\begin{abstract}
In this paper, we present the implementation of a branch-and-cut algorithm for solving Steiner tree problems in graphs. Our algorithm is based on an integer programming formulation for directed graphs and comprises preprocessing, separation algorithms, and primal heuristics. We are able to solve nearly all problem instances discussed in the literature to optimality, including one problem that - to our knowledge - has not yet been solved. We also report on our computational experiences with some very large Steiner tree problems arising from the design of electronic circuits. All test problems are gathered in a newly introduced library called SteinLib that is accessible via the World Wide Web. (C) 1998 John Wiley \& Sons, Inc. Networks 32: 207-232, 1998
\end{abstract}

Keywords: branch-and-cut; cutting planes; reduction methods; Steiner tree; Steiner tree library

\section{INTRODUCTION}

Given an undirected graph $G=(V, E)$ and a node set $T$ $\subseteq V$, a Steiner tree for $T$ in $G$ is a subset $S \subseteq E$ of the edges such that $(V(S), S)$ contains a path from $s$ to $t$ for all $s, t \in T$, where $V(S)$ denotes the set of nodes incident to an edge in $S$. In other words, a Steiner tree is an edge set $S$ that spans $T$. The Steiner tree problem is to find a minimal Steiner tree with respect to some given edge costs $c_{e}, e \in E$. This problem is known to be I $(P$-hard (Karp [28]), even for grid graphs (Garey and Johnson [18]).

Nourished from the increasing demand in the design of electronic circuits, the solution of Steiner tree problems has received considerable and strongly growing attention in the last 20 years. Among the proposed solution methods

Correspondence to: A. Martin

Mathematical Subject Classification (1995): 05C40, 90C06, 90C10, 90C35 are exact algorithms, heuristic procedures, approximation algorithms, polynomial algorithms for special instances, polyhedral approaches, preprocessing techniques, and more. Excellent surveys were given in Winter [40], Maculan [32], Hwang and Richards [25], and Hwang et al. [26]. To solve the Steiner tree problem to optimality, Aneja [1] proposed a row-generation algorithm based on an undirected formulation, Dreyfus and Wagner [11] and Lawler [29] used dynamic programming techniques, Beasley $[4,5]$ presented a Lagrangean relaxation approach, Wong [43] described a dual-ascent method, Lucena [31] combined Lagrangean and polyhedral methods, and Chopra et al. [8] developed a branch-and-cut algorithm. In particular, polyhedral methods have turned out to be quite powerful in finding optimal solutions for various Steiner tree problems. Reasons for that are the better understanding of the associated polyhedra, the availability of fast and robust LP solvers, and the experience gained to turn the theory into an algorithmic tool.

This paper moves within this framework and presents

(C) 1998 John Wiley \& Sons, Inc. 
a branch-and-cut algorithm. It is strongly related to the algorithm described in Chopra et al. [8]; we solve the same integer programming formulation, again by means of the separation of cutting planes. However, the new algorithm differs considerably, not only in several aspects of implementation but also due to some extensions. The main extensions are a more effective preprocessing phase by incorporating three preprocessing tests, an extension of the initial integer program with so-called flow-balance constraints, and a more careful and more efficient separation of active cut constraints resulting in leaner LPs. In Section 2, we review two different integer programming formulations. The second, on which the branch-and-cut algorithm is based, is a bidirected version of the first. In Section 3, we discuss preprocessing and exploit ideas known from the literature. In particular, our presolve algorithm includes three strong reduction techniques of Duin and Volgenant $[13,14]$. Our computational results demonstrate how important preprocessing is: Without this tool, it would not have been possible to solve any of the large instances. Details of the cutting plane phase of our branch-and-cut algorithms are discussed in Section 4. It includes refined separation strategies (resulting in leaner LPs) and improved primal heuristics such that at an earlier stage the lower- and upper-bound values meet. Extensive tests are given in Section 5. We solve almost all test instances from the literature including one problem that to our knowledge - has not yet been solved and find the optimal solution for many very large instances arising from real-world problems in the design of electronic circuits. We introduce a library for Steiner tree problems called SteinLib (including most of the models from the literature and all new VLSI-instances discussed in this paper). This library is available via anonymous $\mathrm{ftp}$ or from WWW at URL: ftp://ftp.zib.de/pub/Packages/mptestdata/steinlib/.

\section{INTEGER PROGRAMMING FORMULATION}

In this section, we present the integer programming formulation that we are going to solve with our branch-andcut algorithm. Let an undirected graph $G=(V, E)$ with edge costs $c_{e} \geq 0, e \in E$, be given. We assume throughout the paper that the edge costs are nonnegative and integer. In addition, there is a node set $T \subseteq V$, called the set of terminals. We will denote an instance of the Steiner tree problem by the triple $\operatorname{ST}(G, T, c)$.

A canonical way to formulate the Steiner tree problem as an integer program is to introduce, for each edge $e$ $\in E$, a variable $x_{e}$, indicating whether $e$ is in the Steiner tree $\left(x_{e}=1\right)$ or not $\left(x_{e}=0\right)$. Consider the integer program $\min c^{T} x$

(i) $\quad x(\delta(W)) \geq 1, \quad$ for all $W \subset V$,

(uSP)

$$
W \cap T \neq \varnothing,
$$

(ii) $0 \leq x_{e} \leq 1, \quad$ for all $e \in E$,

(iii) $x$ integer,

where $\delta(X)$ denotes the cut induced by $X \subseteq V$, that is, the set of edges with one end node in $X$ and one in its complement, and $x(F):=\sum_{e \in F} x_{e}$, for $F \subseteq E$. It is easy to see that there is a one-to-one correspondence between Steiner trees in $G$ and $0 / 1$ vectors satisfying (uSP) (i). Hence, the Steiner tree problem can be solved via (uSP).

Another way to model the Steiner tree problem is to consider the problem in a directed graph. We replace each edge $[u, v] \in E$ by two antiparallel $\operatorname{arcs}(u, v)$ and $(v$, $u)$. Let $A$ denote this set of arcs and $D=(V, A)$, the resulting digraph. We choose some terminal $r \in T$, which will be called the root. A Steiner arborescence (rooted $a t r)$ is a set of $\operatorname{arcs} S \subseteq A$ such that $(V(S), S)$ contains a directed path from $r$ to $t$ for all $t \in T \backslash\{r\}$. Obviously, there is a one-to-one correspondence between (undirected) Steiner trees in $G$ and Steiner arborescences in $D$ which contain at most one of two antiparallel arcs. Thus, if we choose arc costs $\vec{c}_{(u, v)}:=\vec{c}_{(v, u)}:=c_{[u, v]}$, for $[u, v]$ $\in E$, the Steiner tree problem can be solved by finding a minimal Steiner arborescence with respect to $\vec{c}$. Note that there is always an optimal Steiner arborescence which does not contain an arc and its antiparallel counterpart, since $\vec{c} \geq 0$. Introducing variables $y_{a}$ for $a \in A$ with the interpretation that $y_{a}:=1$, if $\operatorname{arc} a$ is in the Steiner arborescence, and $y_{a}:=0$, otherwise, we obtain the integer program

$\min \vec{c}^{T} y$

(i) $y\left(\delta^{+}(W)\right) \geq 1$ for all $W \subset V$, $r \in W$,

(dSP) $(V \backslash W) \cap T \neq \varnothing$,

(ii) $0 \leq y_{a} \leq 1, \quad$ for all $a \in A$,

(iii) $y$ integer,

where $\delta^{+}(X):=\{(u, v) \in A \mid u \in X, v \in V \backslash X\}$ for $X$ $\subset V$, that is, the set of arcs with tail in $X$ and head in its complement. Again, it is easy to see that each $0 / 1$ vector satisfying (dSP) (i) corresponds to a Steiner arborescence, and, conversely, the incidence vector of each Steiner arborescence satisfies (dSP) (i) - (iii). How are the models (uSP) and (dSP) related?

Polyhedral aspects of both models are intensively dis- 


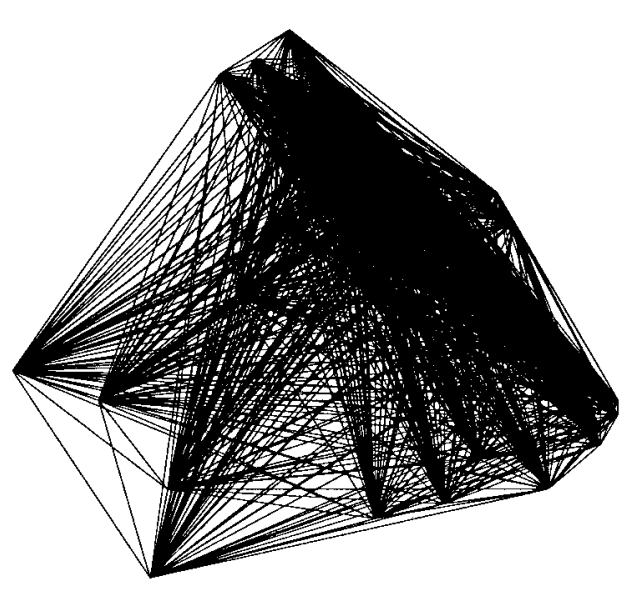

Fig. 1. Original problem.

cussed in the literature. The undirected model was studied in Grötschel and Monma [23], Goemans [19], Goemans and Myung [20], and Chopra and Rao [9, 10], whereas the directed version, in Ball et al. [3], Fischetti [17], Goemans and Myung [20], and Chopra and Rao [9, 10]. Chopra and Rao [9] and Goemans and Myung [20] related both formulations. Chopra and Rao [9] showed that the optimal value of the LP relaxation of the directed model $z_{d}:=\min \left\{\vec{c}^{T} y \mid y\right.$ satisfies (dSP) (i) and (ii) $\}$ is greater or equal to the corresponding value of the undirected formulation $z_{u}:=\min \left\{c^{T} x \mid x\right.$ satisfies (uSP) (i) and (ii) $\}$. Even, if the undirected formulation is tightened by the so-called Steiner partition inequalities (see Grötschel and Monma [23]; Chopra and Rao [9]) and odd hole inequalities (see Chopra and Rao [9]), this relation holds. In addition, Goemans and Myung [20] showed that $z_{d}$ is independent of the choice of the root $r$. These results suggest the directed model and we followed this suggestion. Nevertheless, one disadvantage of the directed model is that the number of variables is doubled. But it will turn out that this is not really a bottleneck, since we are minimizing a nonnegative objective function, and thus the variable of one of two antiparallel arcs will usually be at its lower bound.

It should be mentioned that further models to solve the Steiner tree problem can be found in the literature; for example, models based on flow formulations (Wong [43]; Maculan [32]) or models extending the undirected formulation by introducing node variables (Lucena [31]; Goemans and Myung [20]). Relations between relaxations of these and the above-discussed models can be found in Wong [43], Maculan [32], Duin [12], and Goemans and Myung [20].

\section{PREPROCESSING}

Preprocessing is a very important algorithmic tool in solving combinatorial and integer programming problems of large scale. The idea, in general, is to detect unnecessary information in the problem description and to reduce the size of the problem by logical implications. For the Steiner tree problem, many reduction methods are discussed in the literature and have been shown to be very effective for solving large instances; see, for example, Balakrishnan and Patel [2], Beasley [4], Chopra et al. [8], Duin [12], Duin and Volgenant [14], Lucena [31], Winter [41], and Winter and Smith [42]. These methods focus on detecting special configurations that allow one to neglect certain edges and/or nodes for the optimization or they show that some edges and/or nodes are contained in some optimal solution. In this section, we sketch the main concepts from the literature and show how they are incorporated in our code.

How successful preprocessing methods might be in reducing the size of some problem is demonstrated in Figures 1 and 2. Figure 1 shows the original graph of problem br (complete graph on 58 nodes; for a description of the problem, see Section 5), and Figure 2, the graph that we obtain after applying our preprocessing algorithm.

\subsection{Degree-Test I}

The following tests summarized under the name degreetest I ( see [4]) are easy to check:

(i) A nonterminal node of degree one can be removed.

(ii) If a nonterminal node $v$ is of degree two, node $v$ and the two incident edges $[u, v]$ and $[v, w], u \neq w$, can be replaced by an edge connecting $u$ and $w$ of $\operatorname{cost} c_{[u, w]}=c_{[u, v]}+c_{[v, w]}$.

(iii) An edge incident to a terminal node of degree one is always in an optimal solution.

(iv) If an edge $e$ is of minimal cost among the edges incident to a terminal node, and the other end node is also a terminal, then $e$ is choosable in any optimal solution.

\subsection{Special-Distance-Test}

This test (introduced in Duin and Volgenant [13]) computes for each pair of nodes a number (called the special distance) which can be exploited to remove some edges.

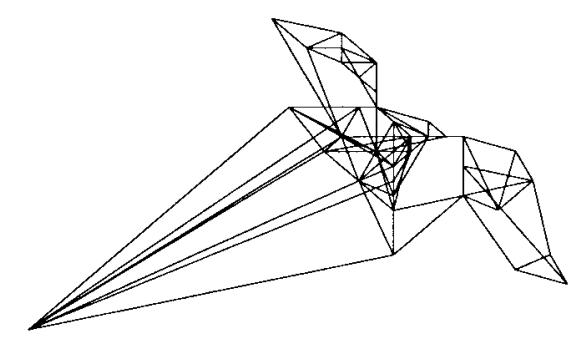

Fig. 2. Reduced problem. 
Definition 3.1 (Special Distance). Let two nodes $u, v$ $\in V$ with $u \neq v$ be given, and consider some path $P \subseteq E$ connecting $u$ and $v$. Set $T_{P}=V(P) \cap T \cup\{u, v\}$ and let

$$
\begin{aligned}
& b(P)=\max \{c(F) \mid F \subseteq P \text { is a path connecting } \\
& \text { two nodes from } T_{P} \text { such that } \\
&\left.\left|T_{P} \cap V(F)\right|=2\right\} .
\end{aligned}
$$

The number

$$
s(u, v)=\min \{b(P) \mid P \text { is a path connecting } u \text { and } v\}
$$

is called the special distance (between $u$ and $v$ ).

To give an idea what $s(u, v)$ means, consider each terminal as a petrol station and suppose you want to drive from location $u$ to $v$. Then, $s(u, v)$ denotes the distance you must be able to drive without refilling if you choose among all possible routes. Note that the following relations

$$
s(u, v) \leq d(u, v) \leq c_{[u, v]}
$$

hold, where $d(u, v)$ denotes the length of a shortest path between $u$ and $v$. The special distance can be computed by a modified shortest path algorithm (cf. Hwang et al. [26]).

Given the values $s(u, v)$ for all $u, v \in V$, there is an easy and very effective test for deleting edges. An optimal solution $S^{*}$ of a Steiner tree problem $\operatorname{ST}(G, T, c)$ cannot contain any edge $[u, v] \in E$ with $s(u, v)<c_{[u, v]}$.

The special-distance-test is a generalization of many other tests known in the literature; this was comprehensively treated in Duin and Volgenant [13]. Concerning implementation, it should be noted that certain special cases of this test can be implemented more efficiently. However, one can also resort to a well-performing approximation of the special-distance-test that runs in $O\left(|V| \log |V|+|E|+|T|^{2}\right.$ ) (cf. Duin [12]).

\subsection{Bottleneck Degree $\boldsymbol{m}$ Test}

The bottleneck degree $m$ test introduced in Duin and Volgenant [14] is the following: Consider some node $v \in N$ with $|\delta(v)|=m$. Let $(W, F)$ be the complete graph on node set $W:=V(\delta(v)) \backslash\{v\}$ with edge costs $\bar{s}_{[u, v]}=s(u$, $v$ ) for $[u, v] \in F$. If, for all subsets $U \subseteq W$ with $|U| \geq 3$,

$$
\bar{s}\left(B^{*}\right) \leq \sum_{u \in U} c_{[v, u]},
$$

where $B^{*}$ is the edge set of a minimal spanning tree in $(W, F)$, holds, node $v$ can be deleted, and for all $u, w$ $\in W, u \neq w$, edge $[u, w]$ with cost $c_{[u, w]}=c_{[u, v]}+c_{[v, w]}$ has to be introduced. (In case of parallel edges, only one edge will be retained.) Of course, this might create many new edges, but, in general, most of these can be eliminated by the special-distance-test.

The running time for this test is $O\left(2^{m} \cdot \gamma\right)$, where $\gamma$ denotes the time for computing a minimal spanning tree. Due to the exponential behavior, we perform this test only for $m \leq 3$. In fact, the bottleneck degree $m$ test generalizes the ones in Section 3.1 (i), where $m=1$, and Section 3.1 (ii), where $m=2$.

\subsection{Terminal-Distance-Test}

In this test, we consider a connected subgraph $H=(W$, $F)$ of $G$ with $T \cap W \neq \varnothing$ and $T \backslash W \neq \varnothing$. Let $e$ $=\operatorname{argmin}_{e^{\prime} \in \delta(W)} c_{e^{\prime}}$ and $f=\operatorname{argmin}_{f^{\prime} \in \delta(W) \backslash\{e\}} c_{f^{\prime}}$ be a shortest and a second shortest edge of the cut induced by $W$.

Edge $e=[u, v]$ with $u \in W$ and $v \in V \backslash W$ is part of some optimal solution of $\operatorname{ST}(G, T, c)$ and can thus be contracted, if

$$
c_{f} \geq d_{u}+c_{e}+d_{v},
$$

with $d_{u}=\min \{d(t, u) \mid t \in T \cap W\}$ and $d_{v}=\min \{d(t$, v) $\mid t \in T \backslash W\}$.

Duin and Volgenant [14] introduced this test and gave an implementation in $O\left(|V|^{3}\right)$ steps. In Duin [12], it is shown that the detection of all edges satisfying the condition of the terminal-distance-test needs only $O\left(|V|^{2}\right)$ steps. Note that the last two tests in Section 3.1 (iii) and (iv) are special cases of the terminal-distance-test. Two other special cases are the $R-R$ aggregation method of Balakrishnan and Patel [2] and the nearest vertex test of Beasley [4].

\subsection{Results}

When it comes to implement these reduction methods, several questions arise: Which of these tests should be implemented? For each single test, should all cases be checked (complete test) which might result in high running times or should one restrict the search to certain promising special cases which might result in an incomplete test? In which order should the methods be called? How often should they be called? Some reduction of one test might give rise to further reductions by some other (already performed) test. These questions were already addressed in Duin and Volgenant [14]. With respect to our algorithm, we should also answer the questions: How much effort and computation time should one spent in the preprocessing phase? At what point is it usually better to switch over to the branch-and-cut phase? We tried to find answers in the following way: First, we implemented all the tests and each test in the complete version. We 
called all these tests consecutively and iterated this process until no more reductions could be found. Of course, this might be very time consuming but for large difficult problems it might be worth to reduce as much as possible (see Section 5). For small and medium-sized problems, the situation is different. Often it did not pay to perform a complete test, but rather to switch to the branch-andcut phase which usually solved the (reduced) problem very fast. We performed many test runs to find a balance between the total running times and the success of the reduction methods. Algorithm 3.2 shows our default selection:

\section{Algorithm 3.2 (Default Presolve)}

(1) Degree-Test I

(2) Special-Distance-Test

(3) Degree-Test I

(4) Terminal-Distance-Test

(5) Special-Distance-Test

(6) Degree-Test I

(7) Special-Distance-Test

(8) Degree-Test I

(9) Return

Note that the bottleneck degree $m$ test is not included in our default strategy. For some difficult instances, however, it pays to use the bottleneck degree $m$ test and iterate all four tests as along as there is some reduction possible. The success of our presolve strategy is illustrated in Section 5 .

\section{IMPLEMENTATION DETAILS}

In this section, we describe the implementation of our branch-and-cut algorithm for solving the Steiner tree problem. We assume that the reader is familiar with the general outline of a branch-and-cut algorithm (see Caprara and Fischetti [7] for a survey). Algorithm 4.1 presents the main steps of such an algorithm:

\section{Algorithm 4.1 (Branch-and-Cut Algorithm)}
(1) Initialization
(2) repeat
(3) select a leaf from the tree and consider the associ- ated LP
(4) repeat (iterate)
(5) solve the LP
(6) call primal heuristics
(7) separate violated inequalities and add them to the LP

(9) branch if necessary, otherwise remove the leaf from the tree

(10) until branch-and-bound tree is empty

(11) print the optimal solution

(12) STOP.

In the Initialization phase, we set up the first LP and initialize the branch-and-bound tree with the root node representing the whole problem. In our case, the starting LP is essentially empty, consisting only of the trivial inequalities (dSP) (ii). We experimented with initial cuts for the first LP by doing a breadth-first search from the root to every other terminal and adding the cuts between nodes of different depth. Although these cuts have disjoint support for each root-terminal pair, only the smaller instances profited from this idea. While the number of cutting plane iterations [i.e., the number of runs through Steps (4)-(8)] needed to solve the problems was always smaller, the effect from initially having a lot of dense inequalities (i.e., inequalities with many nonzero entries) in the LP considerably slows down the whole process.

For solving the linear programs, we used CPLEX*, Versions 4.0.9 and 5.0, a very fast and robust linear programming solver, which features both a primal and dual simplex solver and a primal-dual barrier solver. We used the dual simplex algorithm, since the LPs from one iteration to the next stay dual feasible, when cutting planes are added or variables are fixed to one of their bounds. It turned out that the best pricing strategy was steepestedge pricing, that is, to select a variable entering the basis that has largest (obtuse) angle with the gradient of the objective function. However, for some instances (in particular, for large grid problems), the arising LPs are highly primal and dual-degenerated.

We tried to avoid degeneracy by perturbing the objective function. We used $\tilde{c}=\vec{c}-\mathrm{b} \varepsilon_{a}$, where $b=\min \left(10^{-1}, \frac{1}{2(|A|+1)}\right)$, and $\varepsilon_{a} \in[0,1)$ is some uniformly distributed random number for each $a$ $\in A$. Since we assumed that $c$ is an integer, our choice of $\tilde{c}$ ensures that an optimal solution with $\tilde{c}$ is also optimal for $\vec{c}$. The running times for solving the LPs were always better with the perturbed objective function than with the original. Nevertheless, some of the larger problems continued to show signs of degeneracy. We tried two further ways to remedy degeneracy: First, we perturbed the objective function with $b=0.1$. This, however, requires reoptimization with the original objective function after the problem has been solved for the perturbed objective function. Sometimes, this reoptimization step needed several thousand simplex iterations and we obtained a significant speed up only in very few cases. Second, we tried the

* CPLEX is a registered trademark of ILOG. 
primal-dual barrier solver of the CPLEX package. The barrier code does not suffer from degeneracy, but has to solve each LP from scratch so that, on average, it could not outperform the dual simplex method with our initial perturbation. Thus, our default choice to solve the LPs is to use the perturbed objective function $\tilde{c}$ and apply the dual simplex algorithm with steepest-edge pricing.

\subsection{Branching and Selecting Leafs}

In Step (9), if it is necessary to branch, we use strong branching (Bixby [6]), that is, we determine a set of variables whose LP value is close to 0.5 , perform for each variable of this set a certain number of simplex iterations for the linear program where the variable is set to one or zero, and, finally, select the variable in the set as branching variable that obtained the best increase in the LP value.

We run through the branching tree in a depth-firstsearch fashion. The reasons are that the memory requirements for the whole tree stay small and that we try to find a good primal solution as soon as possible. It almost never happened that our branching tree grew to much. Branching was a rather rare event in our computations anyway (within the time limit and with the default parameter setting branching was necessary only in 37 of 414 cases; see Section 5).

\subsection{Primal Heuristic}

The primal heuristic that we use is basically the one introduced by Takahashi and Matsuyama [38]. The idea of this heuristic is to start from one terminal and connect a terminal by a shortest path that is closest to the starting terminal. The next terminal is chosen among the remaining terminals in such a way that it is closest to the already existing path or subtree in general. This process is continued until all terminals are connected. As edge costs for this heuristic we use $\left(1-x_{e}\right) \cdot c_{e}$ for $e \in E$, if $x$ is the optimal solution of the current LP, that is, we try to prefer those edges that are already chosen in the LP solution. (A slightly different objective function was suggested in Lucena [31] who used as edge costs 0 , if $x_{e}$ $=1$, and $c_{e}$, otherwise; Chopra et al. [8] used the original edge costs, but considered only edges $e$ with positive LP value $x_{e}>0$.) As suggested in Rayward-Smith and Clare [36], we also try to improve the heuristic solution by computing a minimal spanning tree among the chosen nodes and prune nonterminal leaves as long as possible.

A parameter to be specified for this heuristic is the starting terminal. Since running the algorithm for all terminals is usually too time-consuming, we made the following selection: We always try the terminal which gave the best solution so far and try in addition up to 10 randomly selected terminals. The frequency in which the heuristic is called in our code is specified by some parameter (default is every five cutting plane iterations).

In 138 out of 414 test examples, the first call to the heuristic found the optimal solution, and in $90 \%$ of the cases, the gap [(heuristic solution - lower bound)/lower bound] was below $5 \%$.

We also experimented with the Rayward-Smith [35] heuristic. The results are quite promising; however, a main bottleneck is the running time, especially for big problems. The reason is that the heuristic requires all-toall node distances, and due to memory limitations, we must compute these on the fly, so most of the time is spent for calculating shortest paths.

\subsection{Separating Inequalities}

In this section, we start with the description of our separation routines for the cut inequalities (dSP) (i). We first discuss how the generic cut separation works and give an efficient implementation. In the following three subsections, we discuss three improvements on the generic cuts: back cuts, nested cuts, and creep-flow cuts. All these cuts aim at selecting violated cuts that give the most progress in terms of an increase in the lower bound with respect to the running time. We finally present a further class of inequalities, the so-called flow-balance inequalities, that may strengthen the LP relaxation further. All four suggestions can be combined with each other, resulting in 16 possible ways to separate inequalities. Based on some computational tests in the last subsection, we present our final separation strategy.

\subsubsection{Generic Cuts}

It is well known that the separation problem for the cut inequalities (dSP) (i) can be solved by any max-flow algorithm and can thus be solved in polynomial time. We regard the LP solution as capacities in the graph and check, for each $t \in T \backslash\{r\}$, whether the minimal $(r, t)$ cut is less than one. If so, a violated cut inequality is found; otherwise, there is none. We add inequalities only if they are violated by at least some epsilon (default is $\left.10^{-4}\right)$. To determine a minimal $(r, t)$-cut, for all $t$ $\in T \backslash\{r\}$, we must call, in principle, $(|T|-1)$ times a max-flow algorithm. However, Hao and Orlin [24] showed that by a careful implementation of a preflowpush algorithm the time to determine minimal cuts from the root node $r$ to all other nodes is comparable with the time to find a single $(r, t)$-cut. If we use the highest label preflow-push algorithm of Goldberg and Tarjan [21] the overall running time of the Hao-Orlin algorithm to determine a minimal $(r, t)$-cut, for all $t \in T \backslash\{r\}$, is $O\left(|V|^{2}|E|^{1 / 2}\right)$. 


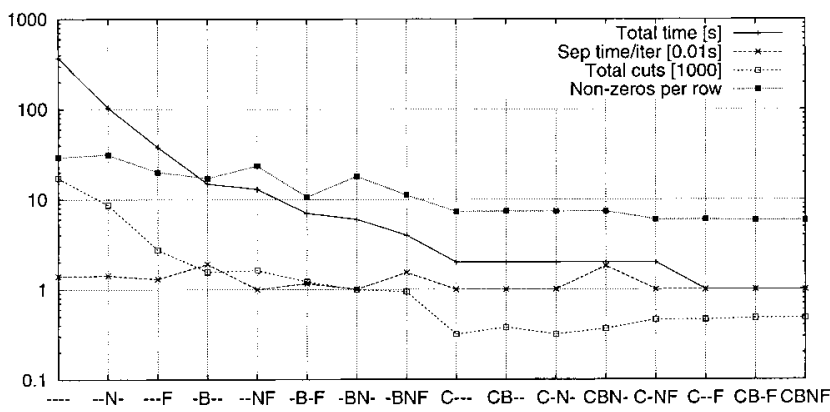

Fig. 3. alue7229.

\subsubsection{Back Cuts}

Chopra et al. [8] described a method to increase the number of separated inequalities by swapping the flow on each arc and checking in addition all $(t, r)$-cuts, for $t$ $\in T \backslash\{r\}$. A drawback here is that we cannot use the speed-up feature mentioned above, since for each $(t, r)$ cut computation, the source node changes and thus the algorithm has to start from scratch again. However, as we will see in Section 4.3.6, this idea significantly improves the overall running time compared with the generic cut generation.

\subsubsection{Nested Cuts}

Another way to increase the number of violated inequalities is to nest the cuts. After finding a minimal cut between $r$ and some terminal $t$, we temporary fix all corresponding variables in the actual LP solution to one and try again to find a cut between $r$ and $t$. We repeat this procedure until the flow between $r$ and $t$ is at least one. This idea can be combined with back cuts so that we are trying to find nested inequalities in both directions. The results of this procedure are usually an increase in the time spent for separation and reoptimization the linear programs per iteration, while the total number of cutting plane iterations drastically decreases, resulting in a total running time of about one magnitude faster than without nested and back cuts.

\subsubsection{Creep-Flow}

We obtained another major speedup in the performance of our algorithm when we implemented the following idea: Instead of trying to increase the number of separated inequalities, we tried to raise the "quality" of the inequalities. Since most of the variables in our LP solution are zero, the optimal solution of the min-cut algorithm is not necessarily arc minimal. So, we add a tiny capacity of some $\epsilon$ (in the code we use $\epsilon=10^{-6}$ ) to all arcs to get among all weight minimal cuts one that is also arc minimal. While this increased the running time for computing a minimal cut, since much more arcs have to be consid- ered, the time needed for reoptimization the linear programs decreased by a factor of 10 . Moreover, the reduction in the number of cutting plane iterations by using these ideas over just adding pure $(r, t)$-cuts is between two and three orders of magnitude.

\subsubsection{Flow-Balance Inequalities}

In our cutting plane phase we take another class of inequalities into consideration. An (optimal) Steiner arborescence can be viewed as a set of flows sending one unit from the root $r$ to each terminal in $T \backslash\{r\}$. This means that for all nonterminal nodes that are not branching nodes in the Steiner arborescence the flow-balance equality $y\left(\delta^{-}(v)\right)=y\left(\delta^{+}(v)\right)$ must hold, and for the other nonterminal nodes, $y\left(\delta^{-}(v)\right) \leq y\left(\delta^{+}(v)\right)$. This is expressed in the following set of inequalities:

$$
\begin{aligned}
& y\left(\delta^{-}(v)\right) \begin{cases}=0, & \text { if } v=r ; \\
=1, & \text { if } v \in T \backslash\{r\} ; \\
\leq 1, & \text { if } v \in N ;\end{cases} \\
& y\left(\delta^{-}(v)\right) \leq y\left(\delta^{+}(v)\right), \quad \text { for } v \in N \text {; } \\
& y\left(\delta^{-}(v)\right) \geq y_{e}, \quad \text { for all } e \in \delta^{+}(v) \text {, } \\
& v \in N \text {. }
\end{aligned}
$$

Note that this system of inequalities is not valid for all Steiner arborescences (e.g., cycles are cut off), but there is always an optimal solution that satisfies these conditions, since the objective function is nonnegative. Note that the addition of inequalities (1) to (dSP) has already been considered in Duin [12]. He gives an example where these inequalities strengthen the LP relaxation.

\subsubsection{A Comparison}

We performed several tests to evaluate the performance of these four separation routines and all its combinations. Figures 3 and 4 show the results of all 16 possible separation strategies for examples alue7229 and taq0631 (for

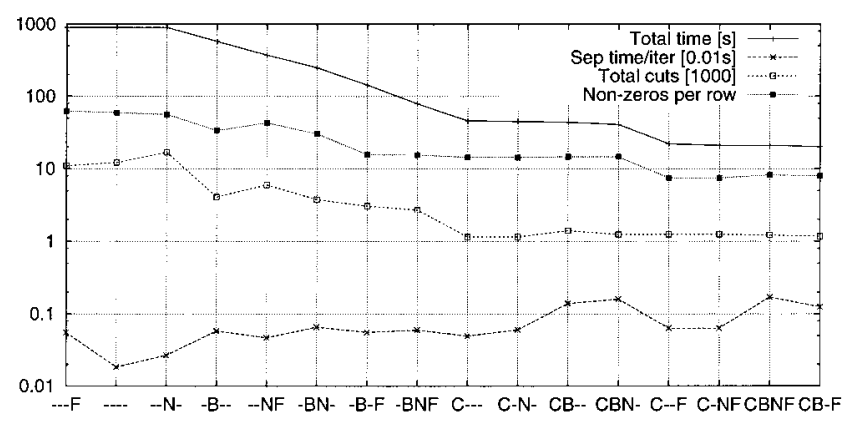

Fig. 4. taq0631. 


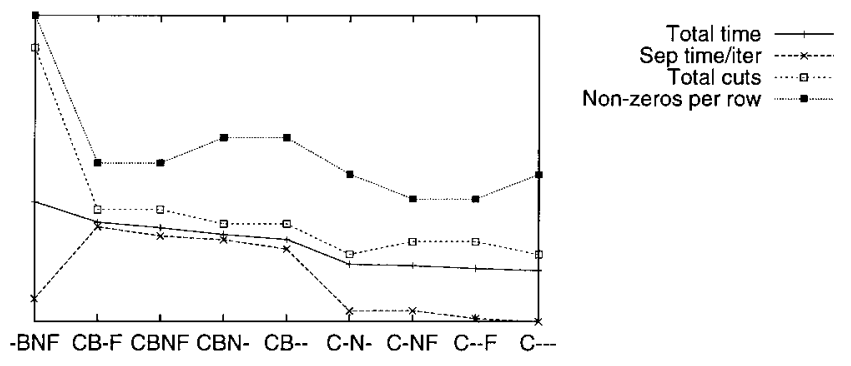

Fig. 5. $g r$.

a description of these problems, see Section 5). $F$ means that flow-balance inequalities are applied; $C, B$, and $N$, indicates that creep-flow cuts, back cuts, and nested cuts are added, respectively; and "-..--' indicates that just the generic cut separation is applied. The $x$-axis shows the 16 possibilities sorted according to their total running time in decreasing order from left to right. The curves depict the total running time (in seconds), the separation time per iteration (in hundredth of seconds), the total number of added cuts (in thousands), and the average number of nonzeros per row. We observe that the differences in the running times are up to two orders of magnitude (note that the $y$ axis is logarithmically scaled). We also see that the strategy " $-\mathrm{B}--$ "' that was used in Chopra et al. [8] gives a significant speed up compared with just adding generic cuts, although the separation time per iteration increases. This was already observed by Chopra et al. However, their strategy is not the overall best. For almost all combinations, it is better to apply flow-balance inequalities. The same holds for creep-flows; the strategy with creep-flows is always better than the one without. The reason is mainly a significant reduction in the number of generated cuts. In both figures, the eight combinations using creep-flows together with "-BNF" are always the best. We evaluated these nine strategies on some larger instances. Figures 5 and 6 show the results for problem $g r$ and $m s m 1234$ (note that the curves are not uniformly and the $y$-axis is not logarithmically scaled any more to better illustrate the differences of the strategies).

The "-BNF-" strategy shows a big increase in the number of cuts and nonzeros resulting in high LP times (LP times are not shown in the diagram). The increase in the LP time per iteration is not completely compensated by the decrease in the total number of iterations, resulting in running times that are not among the best. For larger instances, this effect becomes even clearer. Again, we recognize the positive impact of the flow inequalities. For example, in problem $g r$, the " $\mathrm{C}-\mathrm{-F}$ "' strategy has the best nonzero per row index. In fact, this strategy is very robust: It is always among the four best, while the performance of the other strategies does not seem to be predictable. It is remarkable that the connection of $\mathrm{C}$ with $\mathrm{B}$ and $\mathrm{N}$ (with or without F) does not outperform ' 'C--F."' Therefore, we have chosen the " $\mathrm{C}-\mathrm{-F}$ " option as the final separation strategy in our branch-and-cut algorithm.

\subsection{Removing Inequalities}

Sometimes in the iteration process inequalities become nonbinding, that is, the slack of the inequalities are positive. In these cases the inequality can be removed from the LP without changing its optimal value. Although the inequality can be violated again, it is, in general, a good idea to remove these inequalities in order to keep the LP small. To minimize the occurrences of these reviolated inequalities, we added a "life" counter to each inequality currently in the LP. If the slack of an inequality is nonzero, the counter is decreased; if the slack is zero, it is reset to an initial value (in our implementation 5). If the counter reaches zero, the inequality is removed. This way we are delaying the removal of inequalities to a point where it is more likely that it will never be used again.

\subsection{Reduced Costs and Reduced Set of Variables}

Every time the primal heuristic finds a better solution, we try to fix variables by the reduced-cost criterium. For a discussion on reduced-cost fixing, see for instance Padberg and Rinaldi [34]. With the exception of the class of so-called incidence problems ( see Section 5), this idea has little effect on the performance of our algorithm. Due to the high degeneracy of the LPs, the reduced costs tend to be very small and, thus, the reduced-cost criterium (and possibly also other reduction methods that are based on reduced costs, see Duin [12]) are likely to fail.

Another commonly known idea is to work only on a reduced set of variables by fixing variables temporally to one of its bounds. After the problem has been solved on the reduced set, we check the reduced costs of the temporally fixed variables, add them if necessary to the current set of variables, and reoptimize. Instead of really removing the variables that are fixed from the problem as it is usually done in such a type of column-generation algorithm, we only fix these variables to their bounds and keep them in the LP. CPLEX (the LP solver that we use)

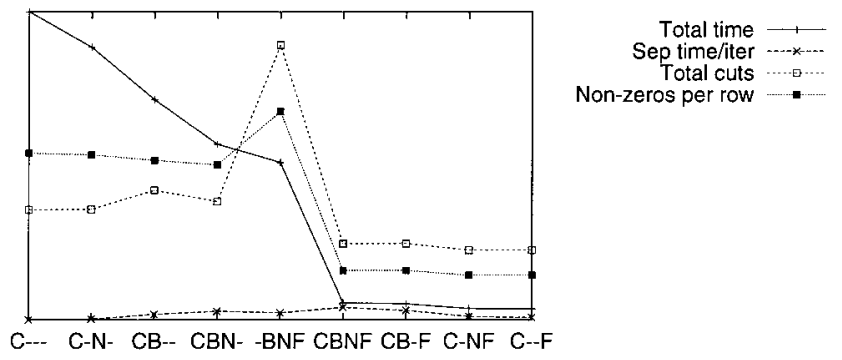

Fig. 6. $m s m 1234$. 
manages fixed variables very efficiently so that we could not detect a major loss of performance (under the assumption that limits of memory are not reached). The advantage is that we do not have to take care of the management of inequalities for which some of the variables are in the current set of variables and some are not. For the limited test runs that we performed for this column-generation idea, we could not obtain a speedup on average.

\section{COMPUTATIONAL RESULTS}

In this section, we report on computational experiences with our branch-and-cut algorithm. Our code is implemented in $\mathrm{C}$ and all runs (with the exception of the incidence problems, see the relevant page in the sequel) are performed on a Sun SPARC 20 Model 71. The test examples include public available benchmarks discussed in the literature, some instances that authors of other Steiner tree codes made us available, and some realistic problems arising in the design of electronic circuits. All instances are gathered in the library SteinLib that is available via anonymous ftp or the World Wide Web.

The format of our tables is as follows: The first column gives the problem name and columns $2-4$ and 5-7 give the number of nodes, edges, and terminals of the original problem and the reduced problem, respectively. Comparing these two sets of columns reflects the success of our preprocessing algorithm. The next three columns give statistics about the branch-and-cut algorithm. Nod contains the number of branch-and-bound nodes ( 1 means that no branching was necessary), Iter gives the number of cutting plane iterations, and Cuts gives the number of violated cuts added to the LP. The following three columns provide information of the root LP, which is the final linear program if no branching was necessary and, otherwise, the last linear program before branching. Frac denotes the number of fractional variables in the root LP, and Rows and $N Z$, the number of rows and nonzeros. Then, time statistics follow: Pre stands for presolve time; $\mathrm{Heu}$, for the heuristic time; and $L P$, for the time spent to solve the LPs; the separation time is shown in column Sep, and, finally, Tot gives the whole running time to solve the problem. The times are in CPU seconds. The time limit for all runs (with the exceptions of e18, diw0234, and some incidence problems) was 10,000 seconds. The last three columns show the solution values. $\mathrm{Heu}(1)$ is the value of the solution found by the first call to the primal heuristic, that is, when no linear programming solution is at hand $(x=0)$. Comparing this value to the lower bound depicted in column $L B$ provides information about the quality of the primal heuristic. If the

\footnotetext{
${ }^{\dagger}$ URL: ftp://ftp.zib.de/pub/Packages/mp-testdata/steinlib.
}

difference between the lower and upper bound is less than 1 , the upper bound in the last column is shown in bold face to indicate that the optimal solution was found. If there is still a gap greater than 1 between $L B$ and $U B$, we have not found the optimal solution within the time limit.

Tables I and II show our results for the test series introduced by Beasley [5]. Test set $\mathrm{C}$ is easy: We solve all instances with one exception within a minute. Interesting to note is that already the first call to the heuristic (without any dual information) gives in 11 out of 20 examples the optimal solution. Series D with 1000 nodes is a bit more difficult: The running times increase up to 6 minutes. However, the optimal solution is obtained in the root node in all but one case ( $d 19)$, that is, branching was not necessary. To solve test series E (with the exception of $e 18$ ), we need up to 2 hours per instance, although still no branching is necessary. The number of cuts needed to solve these examples increases to about 66,000 . We could not detect a correlation between the number of violated inequalities and the number of variables or terminals. The number of inequalities in the final LP is rather high compared with the number of cuts separated. This means that the inequalities mostly stay in the LP whenever they are added and elimination does not happen too often. The exception of test series E is $e 18$. To the best of our knowledge, nobody solved this problem up to now to optimality. We are able to solve it within half a day of CPU time, where Algorithm 3.2 was replaced by a complete reduction test. $e 18$ and $d 19$ are the only examples of Beasley's test set where branching was necessary with the default parameter setting."

Figure 7 depicts a diagram of the run for e16. The $x$-axis shows the number of cutting plane iterations (i.e., the number of LPs that have been solved). There were 267 iterations for example $e 16$ ( see Table II). The curves in the diagram illustrate trends of certain numbers in the course of the algorithm. The top curve Integer gives the number of variables that are integer in the actual LP solution. In the first LP, all variables are integer, since we start just with the trivial inequalities (see above). Thus, the straight line indicates that almost all variables are integer during the whole run of the algorithm (we will see different patterns for other problems in a moment). The next two curves show the upper and lower bounds. The horizontal line for the upper bound means that the first call to the heuristic already found an optimal solution. The lowest curve gives the number of rows in the LP. Its steady increase demonstrates the effect just mentioned that elimination of inequalities from the LP does not frequently happen. This property is common to many test

\footnotetext{
* In fact, branching was only necessary to obtain an optimal solution; the objective function value of the root LP rounded up already yields the optimal solution value.
} 


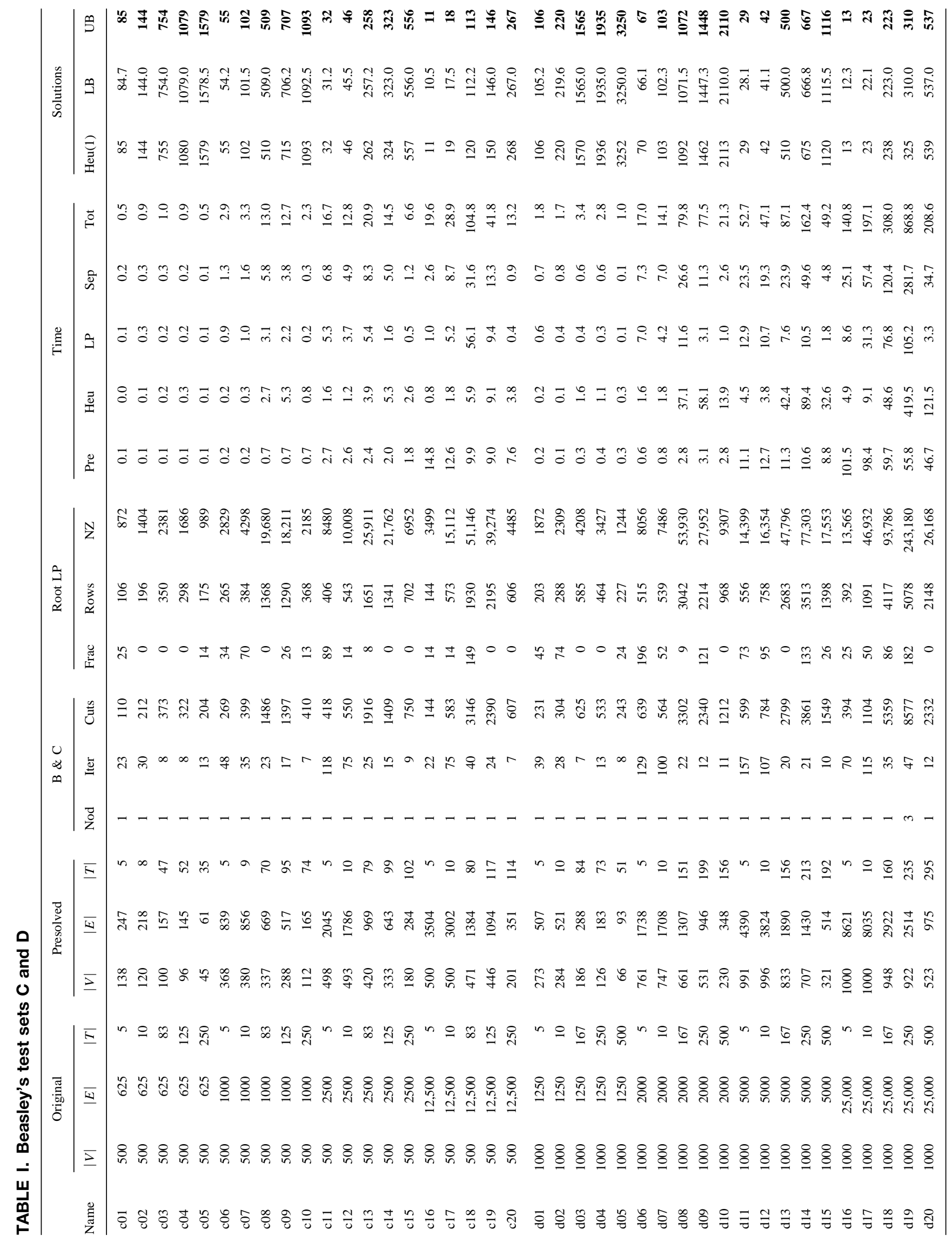




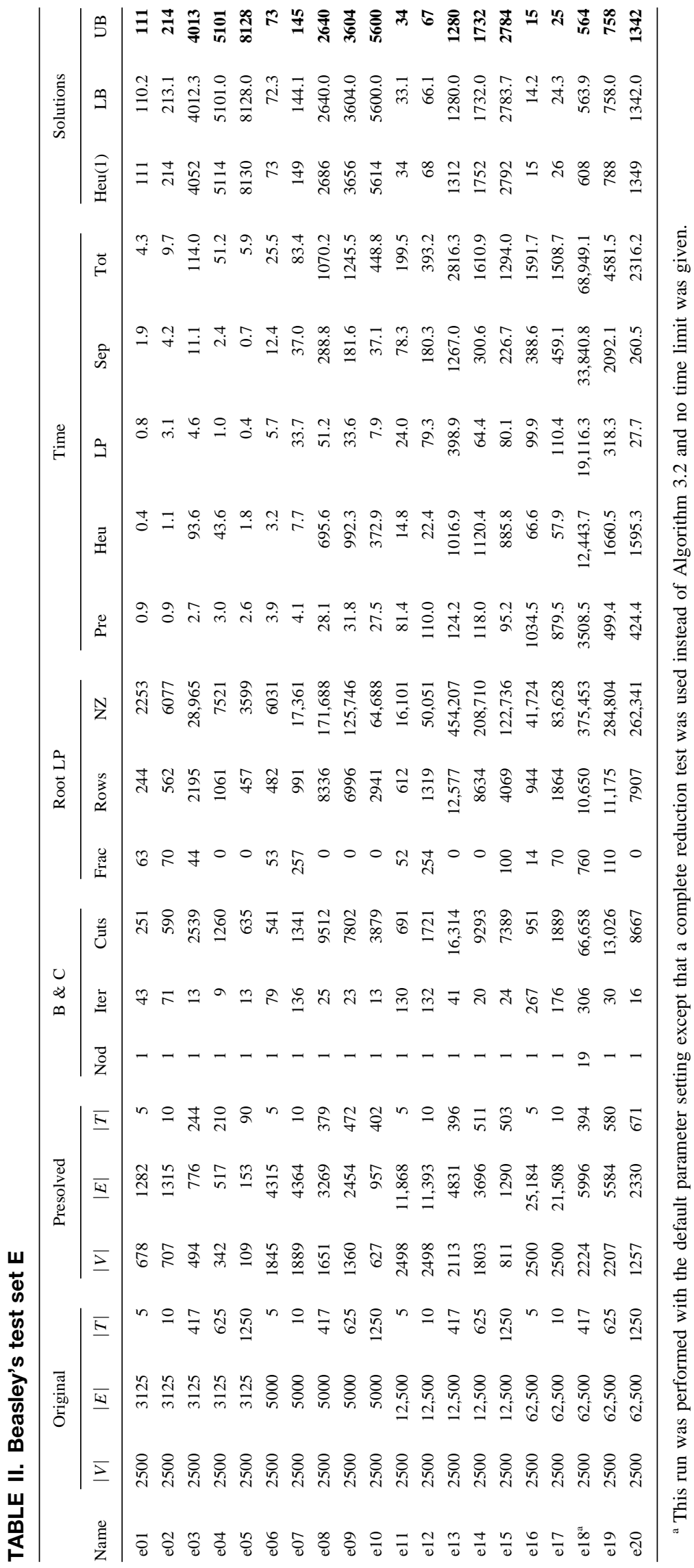




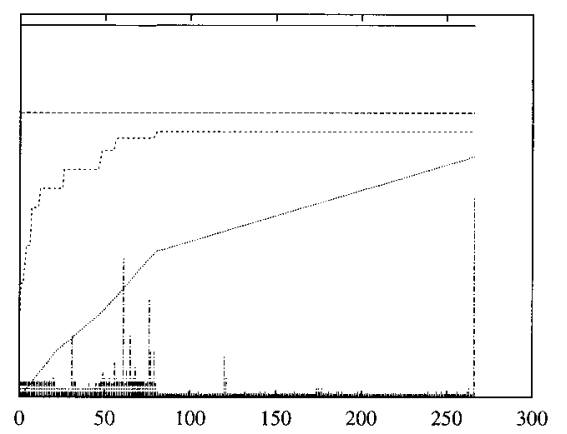

Fig. 7. $e 16$.

examples ( see also Figs. 8-10). The bottom bars depict the number of simplex iterations to solve the LPs; the higher the bars, the more difficult were the LPs to solve. We see that the number of simplex iterations is high if there is an increase in the lower bound, and the numbers are low when there is no progress in the lower bound. We have observed this behavior on many Beasley instances.

Table III contains some instances made us available by Margot [33] and some problems on complete graphs. br was introduced in Ferreira [16], whereas berlin and $g r$ are taken from the TSP library, where some nodes are defined as terminals. It turns out that the winning procedure for complete instances is presolve. Algorithm 3.2 reduces up to $98 \%$ of the edges (variables) and provides the bases for solving even the big $g r$ example with over 200,000 variables within 6 minutes.

The diagram of example $g r$ in Figure 8 shows some different sign patterns from the one of $e 16$. We observe that the number of fractionals (see the curve reflecting the number of Integers) is low at the beginning, increases continuously until the middle of the run, and decreases again toward the end. This $u$-shape behavior is typical for complete instances. We also see that the difficulty of the LPs is correlated to the number of fractional variables, which is also true for the examples depicted in Figures 9 and 10 .
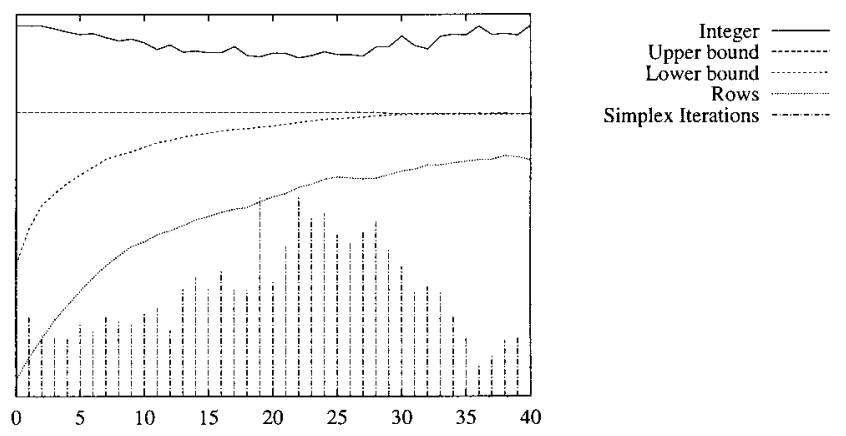

Fig. 8. $g r$.
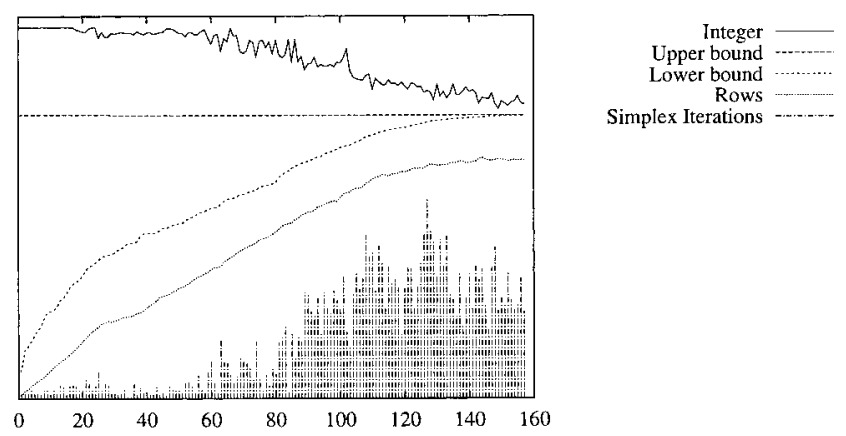

Fig. 9. gap3100.

Table IV contains a collection of examples described in Chopra et al. [8]. E. Gorres made these instances available to us. We solved all these instances within seconds. ${ }^{\S}$ Interesting to note is that almost always the root LP is integer (see Column Frac).

The next series, denoted by $R$, is taken from Soukup and Chow [37] ( see Table V). We solve all of them in about 1 minute. Worthwhile to note are that in 24 of 38 examples the first call to the heuristic already found the optimal solution and that the LP time dominates all other times. The latter fact seems to be typical for grid examples, which the test set $R$ consists of entirely. This phenomenon will become clearer in some of the next tests.

Tables VI and VII show results for the so-called incidence problems obtained from C. Duin. These problems, described in Duin [12] and Duin and Voß [15], are randomly generated and have the following sizes: There are four sizes of the node set $v:=|V|=80,160$, 320, and 640 ; for each of them, 20 variants are generated combining four sizes of the terminal set $|T|=\log v, \sqrt{v}, 2 \sqrt{v}$, and $v / 4$ with five different densities $|E|=(3 v) / 2, v \ln$ $v,[v(v-1)] / 2,2 v$, and $[v(v-1)] / 10$, all values are rounded down to the next integer. Every variant was drawn five times. The problem names have the pattern v.tei, where $v=80,160,320$, and 640 gives the number of nodes of the problem, $t=0,1,2,3$ indicates which of the four alternatives (in the above sequence) of the sizes for the terminal sets have been chosen, $e=0,1,2$, 3, 4 stands for the five densities, and $i=1,2,3,4,5$ distinguishes the five instances drawn for each variant. To give an example, problem 160.141 is the first out of five instances with 160 nodes, $[v(v-1)] / 10=(160 \cdot$ 159) $/ 10=2544$ edges, and $\lfloor\sqrt{v}\rfloor=\lfloor\sqrt{160}\rfloor=12$ terminals. For each variant, our algorithm behaves very similarly for the five instances; thus, we show only the first in Tables VI and VII. The computations for these prob-

\footnotetext{
${ }^{\S}$ The optimal values sometimes differ from the one described in Chopra et al. [8], because they did not add the values of variables fixed by presolve.
} 


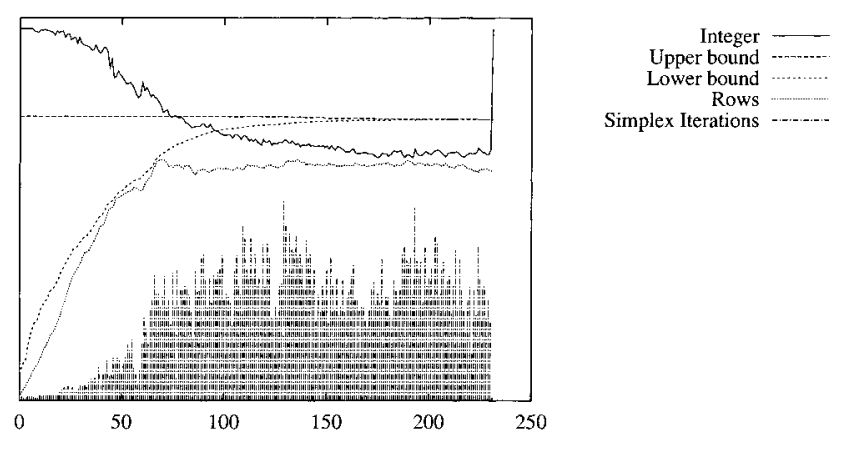

Fig. 10. es40o.

lems were performed on a Sun Enterprise 3000 at a later date, using CPLEX Version 5.0 (instead of Version 4.0.9 as for the other computations ).

The incidence problems show completely different solution characteristics. One main difference is that our presolve algorithm (neither the default nor complete test) could not find any significant reductions (note that the problems were generated with the intention to have difficult problems for presolve, see Duin [12] and Duin and Voß [15]). On the other hand, the examples do not show the same sign of degeneracy and we could fix many variables in the course of the algorithm by the reduced cost criterium (see Section 4.5). To tackle the incident problems, it turned out to be a good idea to restart our branchand-cut algorithm from scratch after a certain percentage of the variables could be fixed. Column $R$ in Tables VI and VII shows the number of restarts performed and Columns 5-7 give, instead of the sizes of the presolved problems (which are almost always identical to the original sizes), the sizes of the problems after the last restart. We see that with this idea of iterative restarts sometimes a significant amount of variables can be fixed, especially if the number of terminals is small (for instance, the number of edges of 640.021 can be reduced by 97\%). We are able to solve all problems on 80 and 160 nodes. However, we have difficulties to solve some of the larger instances. There are problems like 320.311 or 640.141 that we even cannot solve within 1 day. Table VII presents the results, where we used a unique time limit of 10,000 seconds for the difficult instances. Within this time limit, we can give a solution guarantee of at least $4.2 \%$ for all incidence problems.

What one would like to have at this point is a comparison with other codes. However, this is very difficult. People have different machines with different storage spaces, use different packages for the solution of subproblems like linear programs, and so on. We refrain from giving a comparison here. The interested reader may refer to Beasley [5], Chopra et al. [8], Duin [12], or Lucena [31], who developed comparable codes for the Steiner tree problem in graphs.

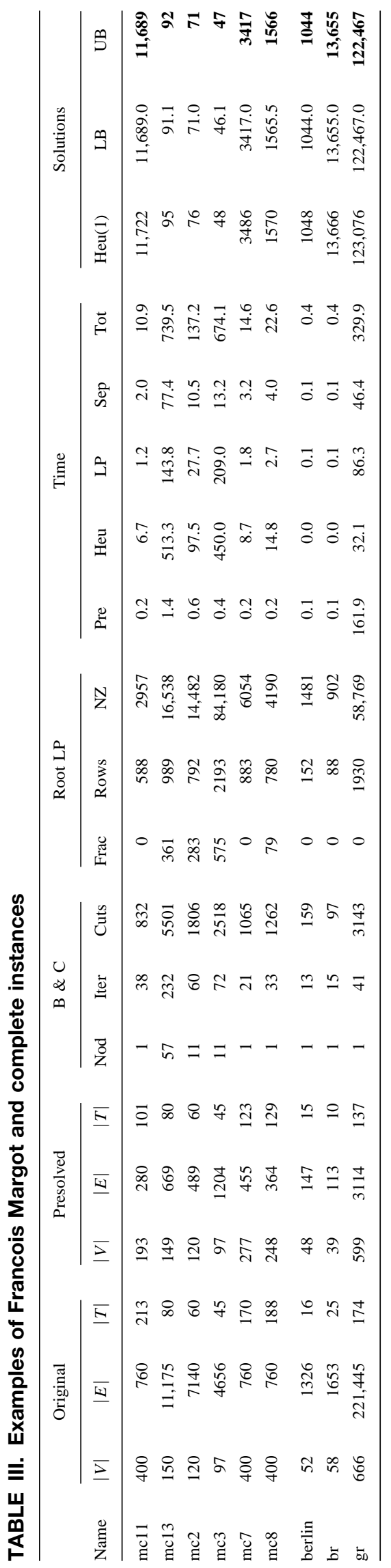




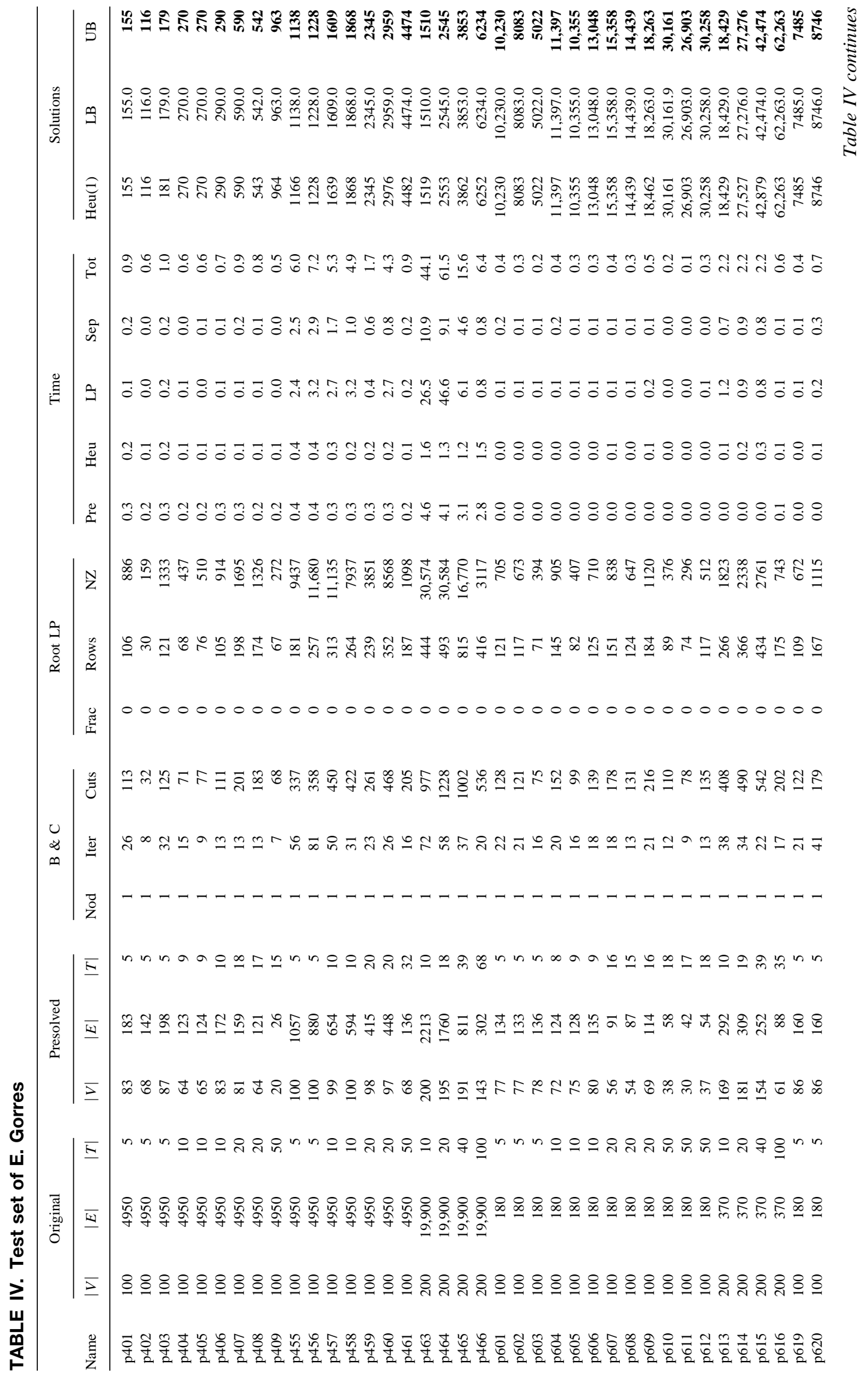




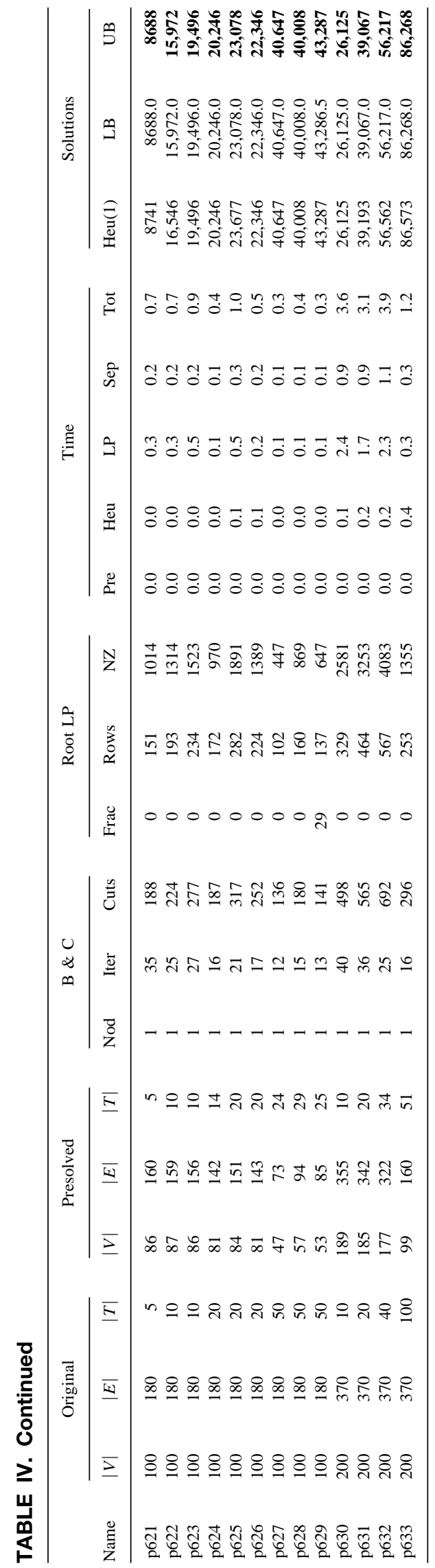

Tables VIII-X give computational results on realworld VLSI instances. One of the challenging problems in the design of electronic circuits is the routing problem, which is, roughly speaking, the task to connect terminal sets via wires on a predefined area. Depending on the underlying technology and the design rules, subproblems arise that can be formulated as the problem of packing Steiner trees in certain graphs (see Lengauer [30] for an excellent treatment of this subject). The problems that we are going to consider result from seven different circuits described in Jünger et al. [27]. The underlying graphs are grid graphs that contain holes. The holes result from so-called cells that block certain areas of the grid. The sets of terminals are located on the border of these holes. For each of the seven circuits and for each terminal set $T_{i}$ (where index $i$ runs from 1 to the number of terminal sets of the circuit), we constructed an instance of the Steiner tree problem. For the graph $G$, we have chosen the underlying grid graph restricted to the minimal enclosing rectangle of the terminal set. The distance of two neighbored grid points in horizontal and vertical directions differ for these circuits. This results in different edge costs for horizontal and vertical edges in $G$.

In the library SteinLib, we put all instances with terminal sets whose cardinality is at least 10 (in total 475). The examples are distinguished by the name of the circuit followed by the index of the terminal set. For example, msm1234 means that the instance is defined by terminal set 1234 of circuit msm. As test problems for our algorithm, we chose for each circuit all instances whose two leading nonzeros of the index of the terminal set differ from the two leading nonzeros of all other indices. If there are more than one index with the same two leading nonzeros, we chose the instance with the smallest index (for instance, among examples msm3727, msm3731, $m s m 3761$, and $m s m 3786$, we chose $m s m 3727)$. In addition, we added an instance with the smallest and largest number of terminals for each circuit. This way we obtained 116 different VLSI test instances.

The success of our branch-and-cut algorithm is shown in Tables VIII-X. We solve 83 out of the 116 instances to optimality within 10,000 seconds and provide a solution guarantee [( upper bound - lower bound)/lower bound] of less than $10 \%$ for $85 \%$ of the examples. The biggest with respect to number of terminals that we solve within the time limit are alue 5067 and alue6735 with 68 terminals each. The biggest in size of the number of edges is $m s m 3727$ with over 8000 edges. However, there are also smaller instances, for example, diw0795 with 10 terminals or $m s m 2601$ with less than 5000 variables after presolve, that we do not solve within the time limit. All runs were performed with the default strategy (except for diw0234 and alut2625); in particular, we applied Algorithm 3.2 to reduce the problem and did not perform a complete reduction test (see Section 3). If there is no time 


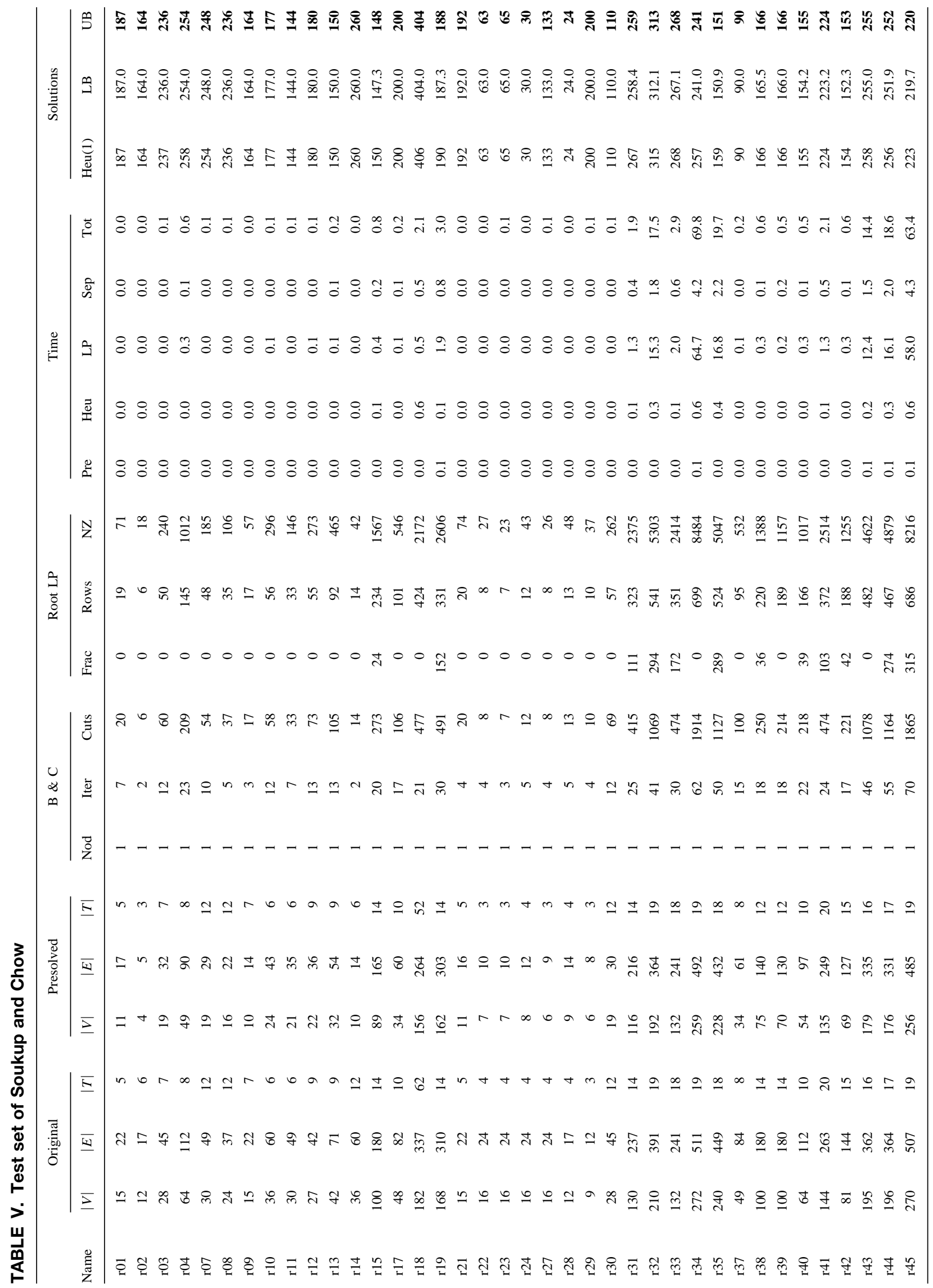




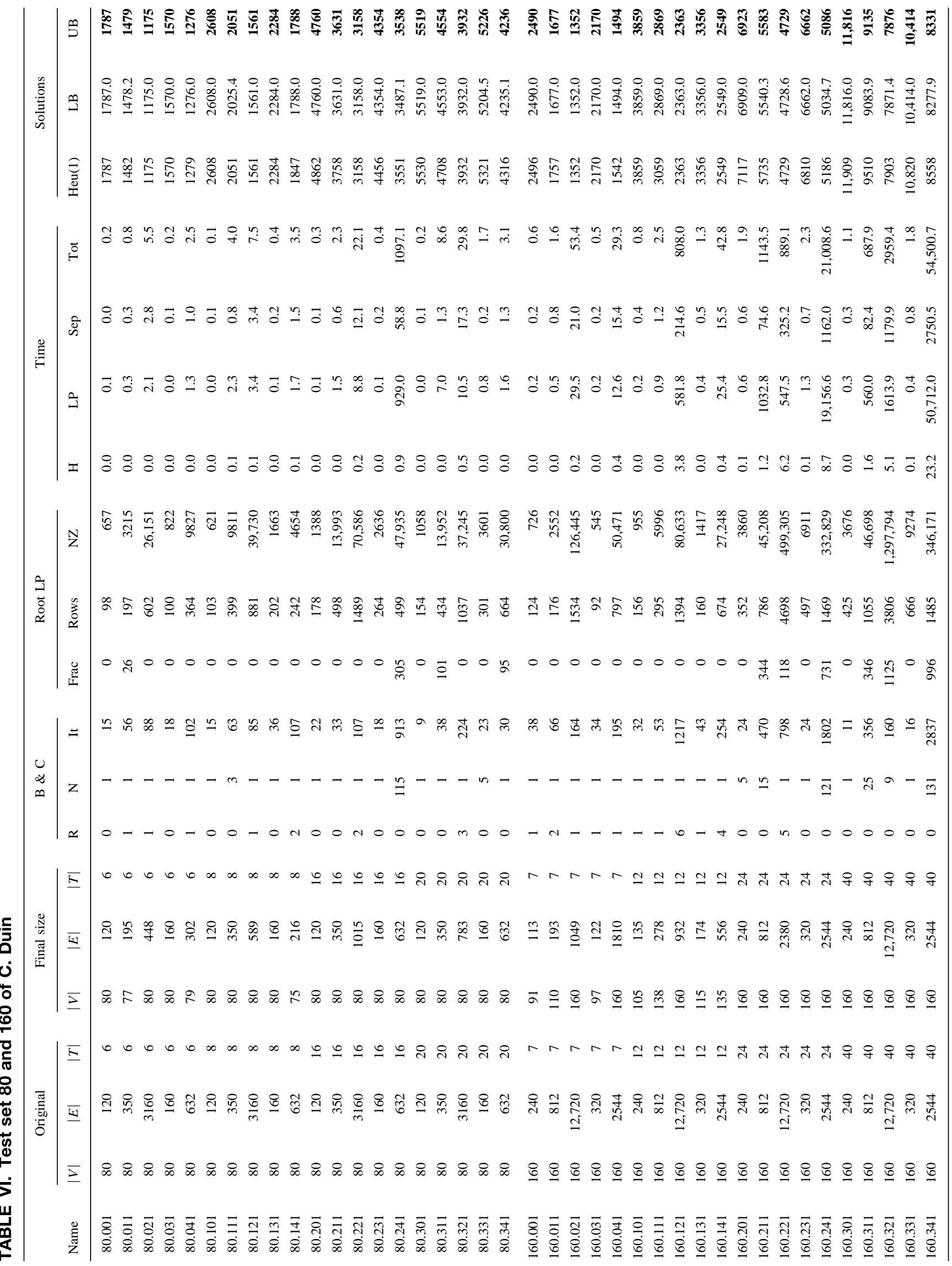




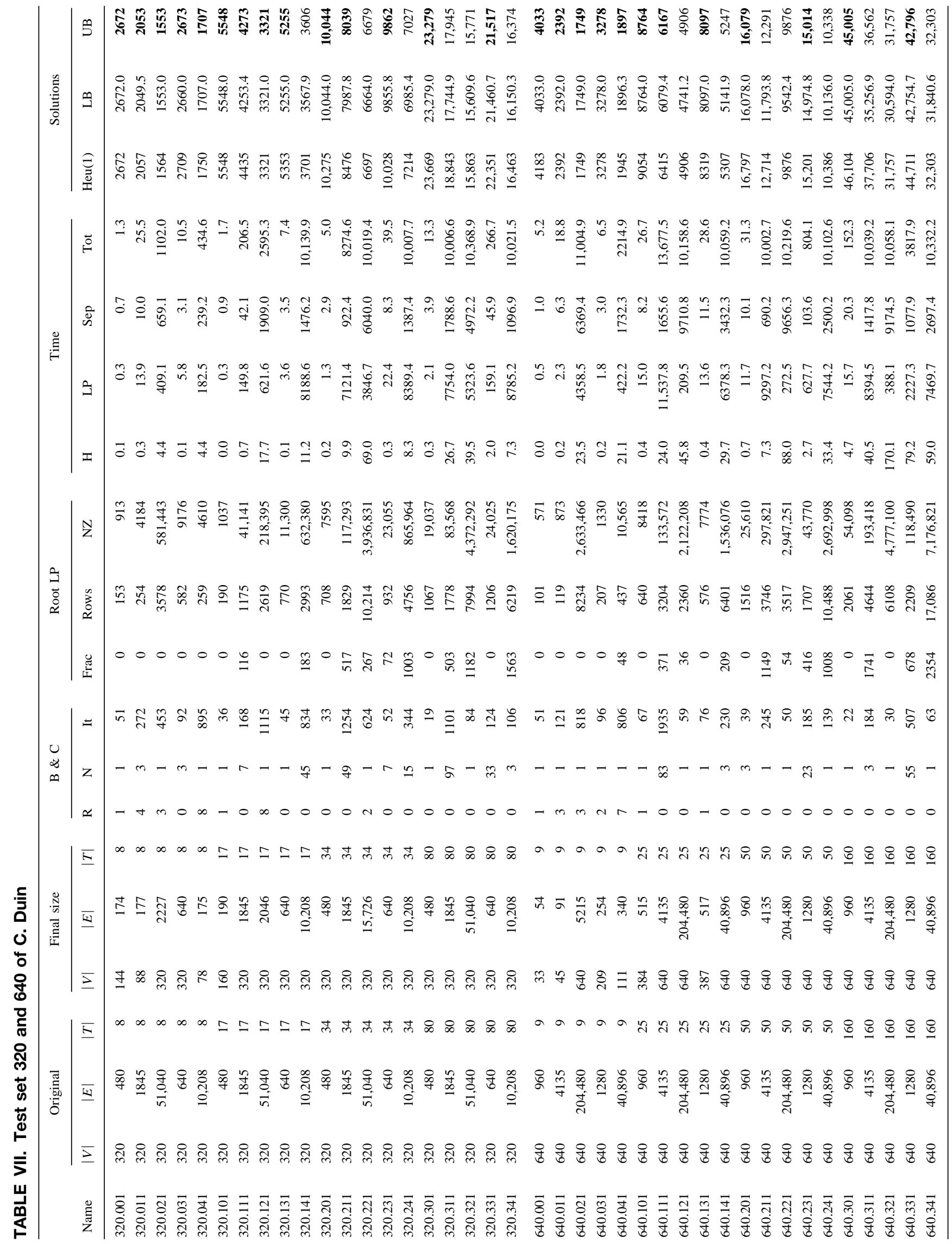




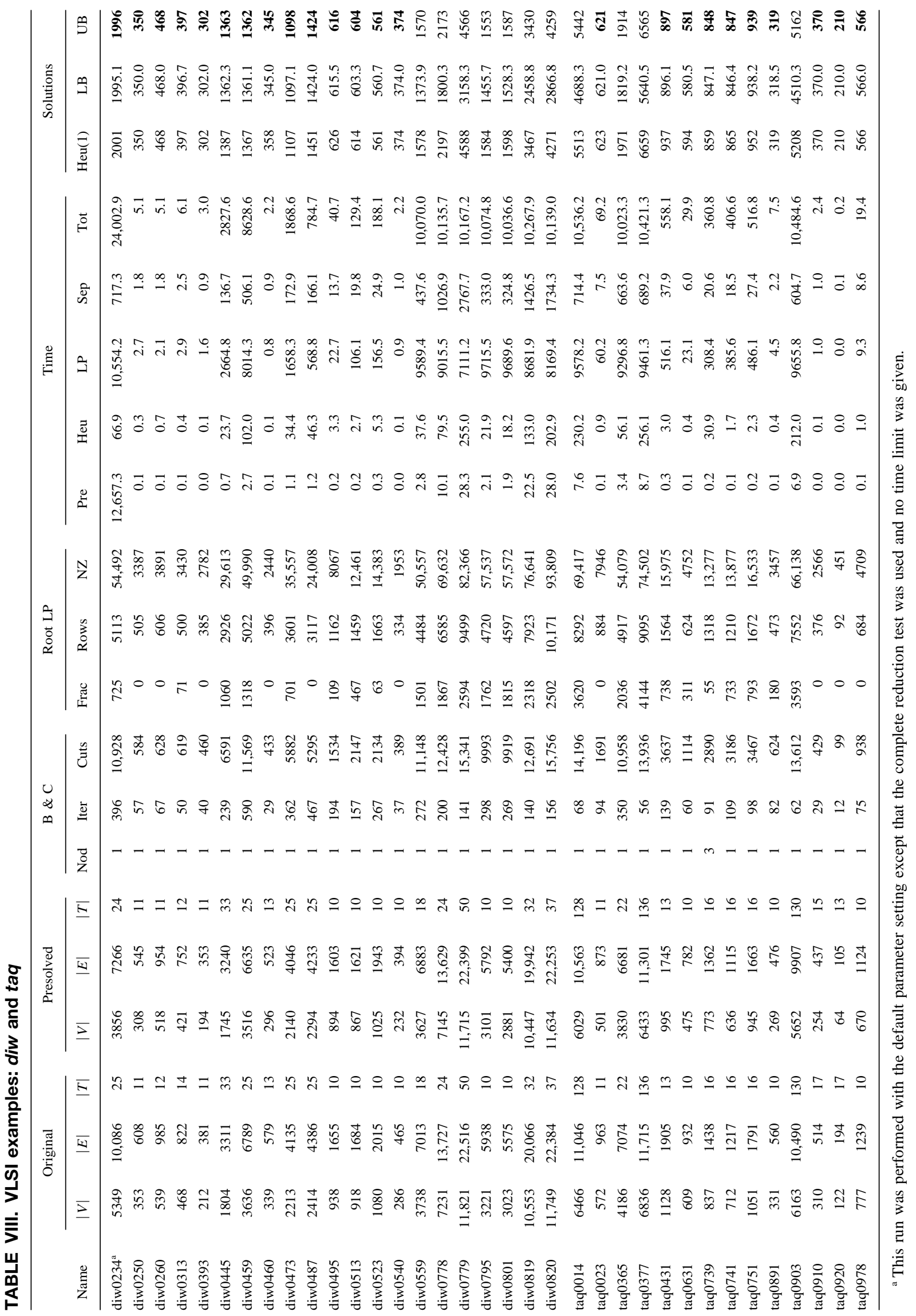




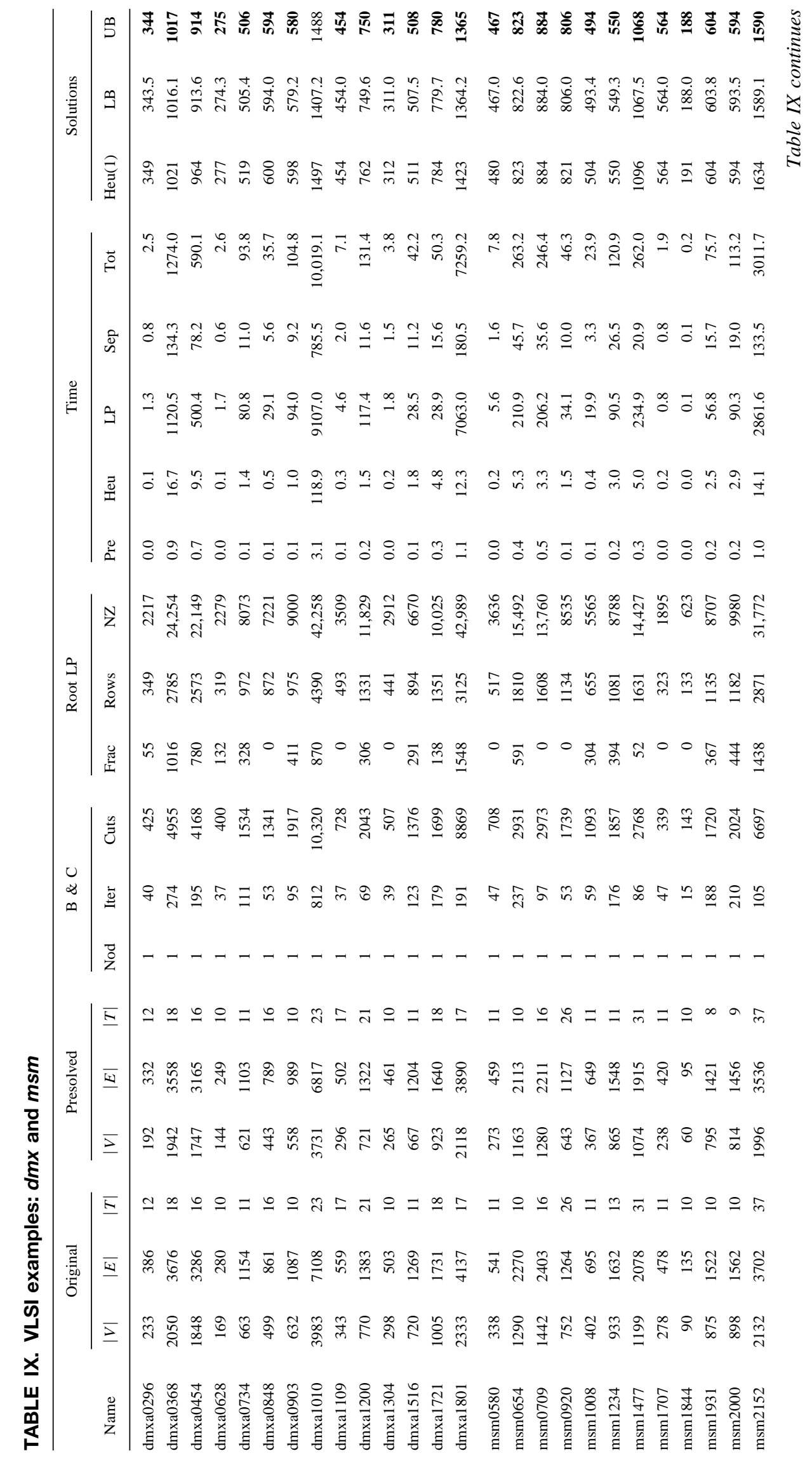




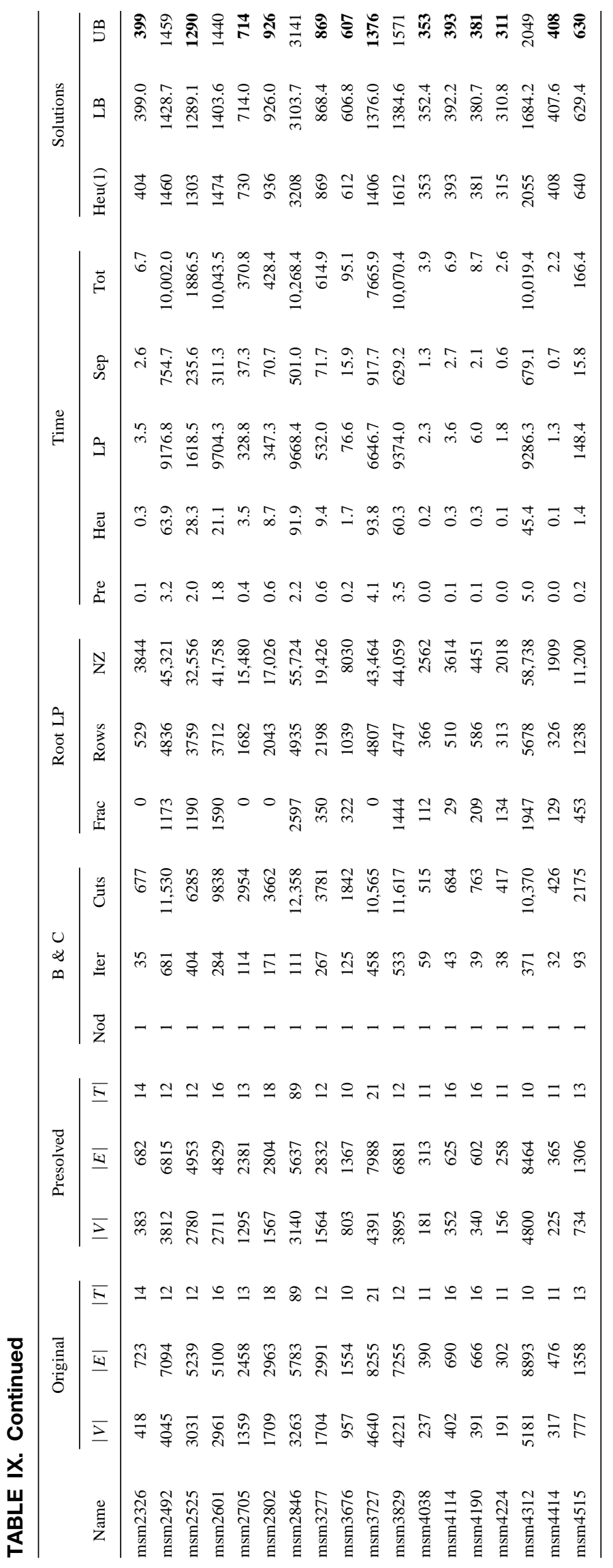




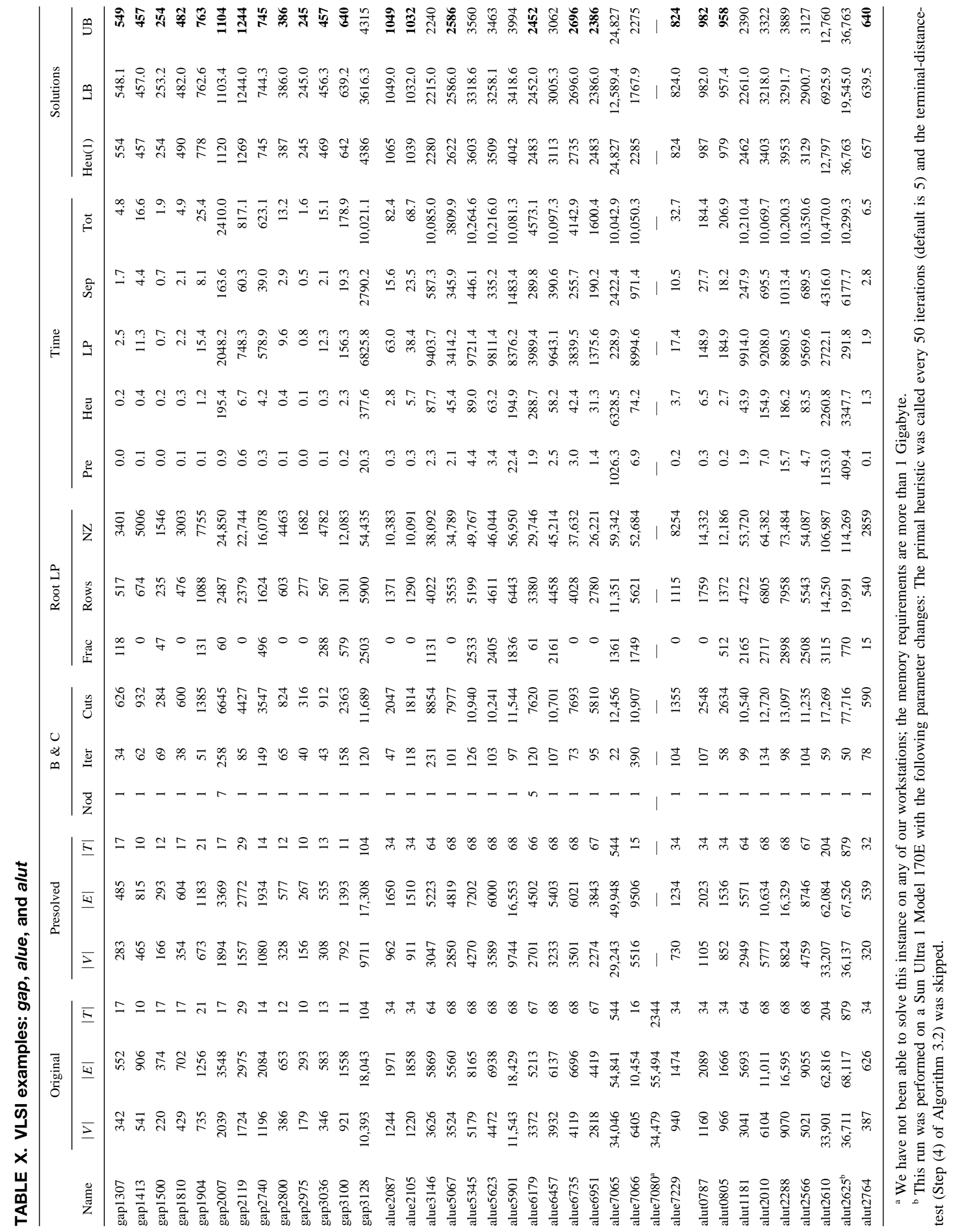




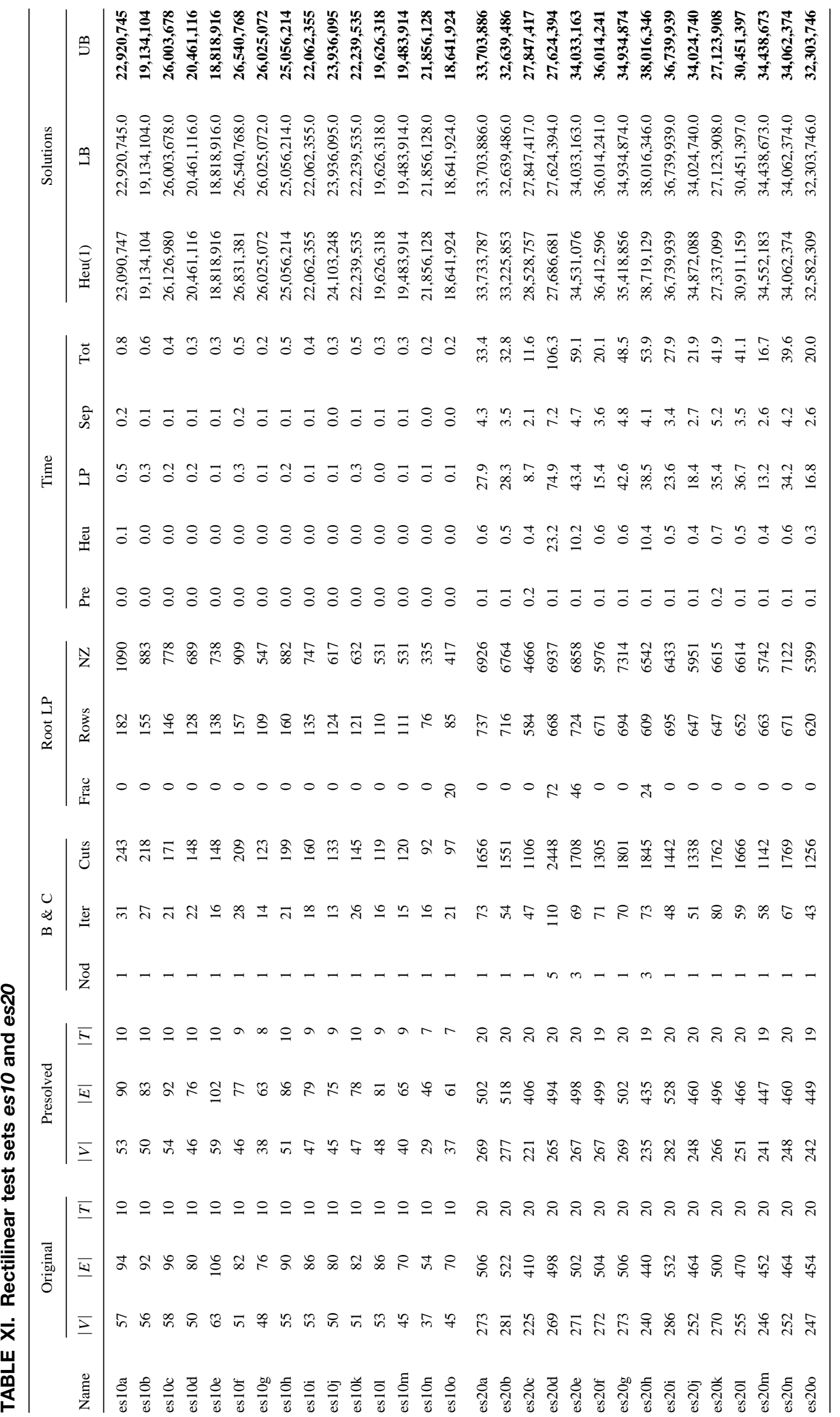




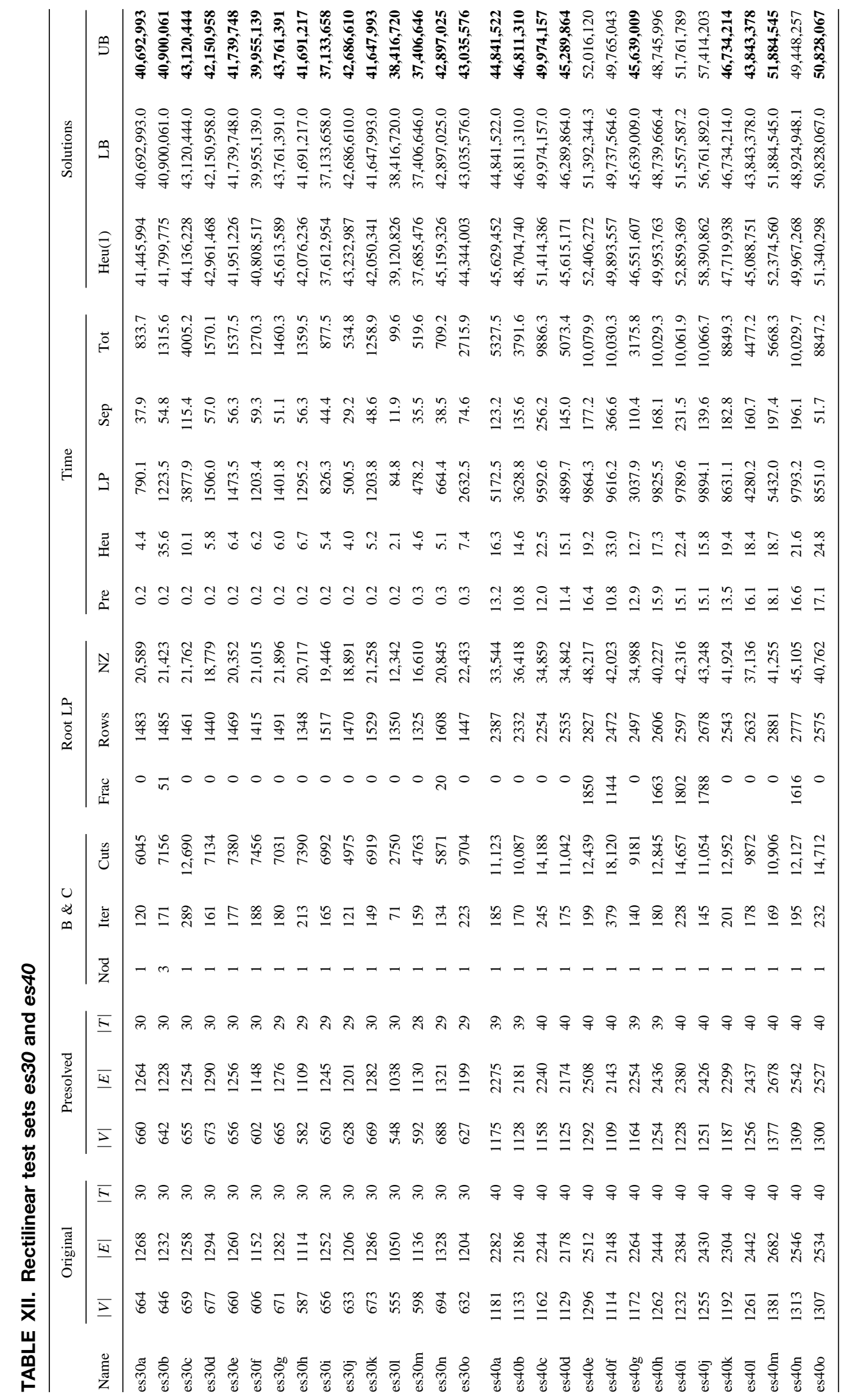


limit given, it usually pays to call all reduction methods to reduce the problem as much as possible in size. For instance, we solve example diw0234 with over 10,000 variables in about 24,000 seconds. The complete presolve reduces the problem from 10,086 edges to 7266, whereas Algorithm 3.2 reduces it just to 9991 edges. However, over 12,000 seconds are spent in presolve when the complete reduction test is performed and only 6 seconds when Algorithm 3.2 is applied. (With the default parameter setting, we obtain after 10,000 seconds an upper bound of 1997 and a lower bound of 1967 providing a solution guarantee of $1.5 \%$.) The difficulty of the VLSI problems seem not only depend on the number of terminals, but also on the shape of the grid graphs, how many holes are there, and how big these holes are. Figure 9 shows a typical diagram for these problems. The numbers of fractional variables continuously increase (see the decrease of curve Integer ), and the LPs get more and more difficult during the runs (see the number of simplex iterations).

Although our code was originally designed for solving Steiner tree problems in graphs, it is, of course, also possible to solve rectilinear instances by modeling them as graph problems. Tables XI and XII show results on rectilinear problems. Table XI contains the examples from Beasley with 10 and 20 terminals. They are not very difficult (up to 4 minutes), although branching is necessary in three cases. However, the situation changes for test sets es 30 and es 40 . The running times rapidly increase with the number of terminals and we are not able to solve all instances with 40 terminals within 10,000 seconds. Our diagram, for example, es $40 o$ in Figure 10, shows that the LPs become increasingly difficult during the run of the program, a behavior that we have already detected to some extent for the VLSI examples. In fact, the LPs are highly dual and primal degenerated, a phenomenon that seems to be inherent for grid problems ( see also Grötschel et al. [22]). Another drawback is that our presolve procedures do not perform well. Reduction methods (as proposed for instance by Winter [41]) that exploit the structure of grid graphs would probably help to solve these instances faster. Recently, Warme [39] proposed an algorithm for rectilinear Steiner tree problems. By exploiting the typical structure of rectilinear problems, he was able to solve much bigger instances in less time.

\section{CONCLUSIONS}

We have presented an implementation of a branch-andcut algorithm for the Steiner tree problem in graphs. We are able to solve almost all instances discussed in the literature. Our algorithm especially performs well on complete and sparse graphs. Here, a good presolve seems to pay. We have also introduced new real-world VLSI instances. We solve many of these instances and provide reasonable solution guarantees (in general, below 15\%) for all examples except for the really big ones with several hundred terminals and tens of thousands of edges. On rectilinear Steiner tree problems, our code performs well only for examples with a small number of terminals. To be competitive with state-of-the-art software for rectilinear problems, our reduction methods have to be adapted to rectilinear instances and more investigations are necessary to avoid degenerated linear programs. All examples discussed in this paper are gathered in a newly introduced library called SteinLib that is accessible via anonymous ftp or the World Wide Web.

\section{REFERENCES}

[1] Y. Aneja, An integer programming approach to the Steiner problem in graphs. Networks 10 (1980) 167178.

[2] A. Balakrishnan and N. Patel, Problem reduction methods and a tree generation algorithm for the Steiner network problem. Networks 17 (1987) 65-85.

[3] M. Ball, W. Liu, and W. Pulleyblank, Two terminal Steiner tree polyhedra. Contributions to Operations Research and Economics - The Twentieth Anniversary of CORE (B. Cornet and H. Tulkens, eds.). MIT Press, Cambridge, MA (1989) 251-284.

[4] J. Beasley, An algorithm for the Steiner problem in graphs. Networks 14 (1984) 147-159.

[5] J. Beasley, An SST-based algorithm for the Steiner problem in graphs. Networks 19 (1989) 1-16.

[6] R. Bixby, Personal communication (1996).

[7] A. Caprara and M. Fischetti, Branch-and-cut algorithms. Annotated Bibliographies in Combinatorial Optimization (M. Dell'Amico, F. Maffioli, and S. Martello, Eds.). Wiley, Chichester (1997) 45-63.

[8] S. Chopra, E. Gorres, and M. R. Rao, Solving a Steiner tree problem on a graph using branch and cut. ORSA J. Comput. 4 (1992) 320-335.

[9] S. Chopra and M. R. Rao, The Steiner tree problem I: Formulations, compositions and extension of facets. Math. Program. 64 (1994) 209-229.

[10] S. Chopra and M. R. Rao, The Steiner tree problem II: Properties and classes of facets. Math. Program. 64 (1994) 231-246.

[11] S. E. Dreyfus and R. A. Wagner, The Steiner problem in graphs. Networks 1 (1971) 195-207.

[12] C. Duin, Steiner's problem in graphs. PhD thesis, University of Amsterdam (1993).

[13] C. Duin and A. Volgenant, An edge elimination test for the Steiner problem in graphs. Oper. Res. Lett. 8 (1989) 79-83.

[14] C. Duin and A. Volgenant, Reduction tests for the Steiner problem in graphs. Networks 19 (1989) 549567. 
[15] C. Duin and S. Voß, Efficient path and vertex exchange in Steiner tree algorithms. Networks 29 (1997) 89-105.

[16] C. E. Ferreira, O problema de Steiner em grafos: Uma abordagem poliédrica. Master's thesis, Universidade de São Paulo (1989).

[17] M. Fischetti, Facets of two Steiner arborescence polyhedra. Math. Program. 51 (1991) 401-419.

[18] M. R. Garey and D. S. Johnson, The rectilinear Steiner tree problem is np-complete. SIAM J. Appl. Math. 32 (1977) 826-834.

[19] M. X. Goemans, The Steiner tree polytope and related polyhedra. Math. Program. 63 (1994) 157-182.

[20] M. X. Goemans and Y. Myung, A catalog of Steiner tree formulations. Networks 23 (1993) 19-28.

[21] A. Goldberg and R. Tarjan, A new approach to the maximum flow problem. J. ACM 35 (1988) 921-940.

[22] M. Grötschel, A. Martin, and R. Weismantel, Packing Steiner trees: A cutting plane algorithm and computational results. Math. Program. 72 (1996) 125-145.

[23] M. Grötschel and C. L. Monma, Integer polyhedra associated with certain network design problems with connectivity constraints. SIAM J. Discr. Math. 3 (1990) $502-523$

[24] J. Hao and J. B. Orlin, A faster algorithm for finding the minimum cut in a graph. Proceedings of the Third Annual ACM-Siam Symposium on Discrete Algorithms, Orlando, FL (1992) 165-174.

[25] F. K. Hwang and D. S. Richards, Steiner tree problems. Networks 22 (1992) 55-89.

[26] F. K. Hwang, D. S. Richards, and P. Winter, The Steiner Tree Problem. Annals of Discrete Mathematics 53, North-Holland, Amsterdam (1992).

[27] M. Jünger, A. Martin, G. Reinelt, and R. Weismantel, Quadratic 0/1 optimization and a decomposition approach for the placement of electronic circuits. Math. Program. 63 (1994) 257-279.

[28] R. M. Karp, Reducibility among combinatorial problems. Complexity of Computer Computations (R. E. Miller and J. W. Thatcher, Eds.), Plenum Press, New York (1972) 85-103.
[29] E. L. Lawler, Combinatorial Optimization: Networks and Matroids. Holt, Rinehart and Winston, New York (1976).

[30] T. Lengauer, Combinatorial Algorithms for Integrated Circuit Layout. Wiley, Chichester (1990).

[31] A. Lucena, Tight bounds for the Steiner problem in graphs. Preprint, IRC for Process Systems Engineering, Imperial College, London (1993).

[32] N. Maculan, The Steiner problem in graphs. Ann. Discr. Math. 31 (1987) 185-212.

[33] F. Margot, Personal communication (1994).

[34] M. Padberg and G. Rinaldi, A branch and cut algorithm for the resolution of large-scale symmetric traveling salesman problems. SIAM Review 33 (1991) 60100.

[35] V. J. Rayward-Smith, The computation of nearly minimal Steiner trees in graphs. Int. J. Math. Educ. Sci. Technol. 14 (1983) 15-23.

[36] V. J. Rayward-Smith and A. Clare, On finding Steiner vertices. Networks 16 (1986) 283-294.

[37] J. Soukup and W. F. Chow, Set of test problems for the minimum length connection networks. ACM/SIGMAP Newslett. 15 (1973) 48-51.

[38] H. Takahashi and A. Matsuyama, An approximate solution for the Steiner problem in graphs. Math. Jpn. 24 (1980) 573-577.

[39] D. M. Warme, A new exact algorithm for rectilinear Steiner trees. Technical report, Telenex Corporation, Springfield, VA 22153 (1997).

[40] P. Winter, Steiner problems in networks: A survey. Networks 17 (1987) 129-167.

[41] P. Winter, Reductions for the rectilinear Steiner tree problem. Research Report 11-95, Rutcors University (1995).

[42] P. Winter and J. M. Smith, Path-distance heuristics for the Steiner problem in undirected networks. Algorithmica 7 (1992) 309-327.

[43] R. T. Wong, A dual ascent approach for Steiner tree problems on a directed graph. Math. Program. 28 (1984) 271-287. 